\title{
EchoGéo
}

$51 \mid 2020$

Territoires « cyclonés »

\section{Activités cycloniques et nouveaux risques dans le golfe d'Aden}

Exemple des impacts socio-économiques du cyclone Sagar sur Djibouti en mai 2018

Jean-Philippe Cherel, Bouh Omar Ali, Moustapha Nour Ayeh et Freddy Vinet

\section{OpenEdition}

Journals

Édition électronique

URL : https://journals.openedition.org/echogeo/18859

DOI : 10.4000/echogeo.18859

ISSN : 1963-1197

Éditeur

Pôle de recherche pour l'organisation et la diffusion de l'information géographique (CNRS UMR 8586)

Référence électronique

Jean-Philippe Cherel, Bouh Omar Ali, Moustapha Nour Ayeh et Freddy Vinet, « Activités cycloniques et nouveaux risques dans le golfe d'Aden », EchoGéo [En ligne], 51 | 2020, mis en ligne le 15 avril 2020, consulté le 10 août 2021. URL : http://journals.openedition.org/echogeo/18859 ; DOI : https://doi.org/ 10.4000/echogeo.18859

Ce document a été généré automatiquement le 10 août 2021.

EchoGéo est mis à disposition selon les termes de la licence Creative Commons Attribution - Pas d'Utilisation Commerciale - Pas de Modification 4.0 International (CC BY-NC-ND) 


\section{Activités cycloniques et nouveaux risques dans le golfe d'Aden}

Exemple des impacts socio-économiques du cyclone Sagar sur Djibouti en mai 2018

Jean-Philippe Cherel, Bouh Omar Ali, Moustapha Nour Ayeh et Freddy Vinet

\section{Introduction}

1 Les cyclones tropicaux sont indubitablement des phénomènes climatiques extrêmes, les plus puissants d'entre eux étant responsables de véritables hécatombes (Bhola: 500000 victimes au Bangladesh en 1970, Nina: 300000 victimes en Chine en 1975, Nargis : 138000 victimes en Birmanie en 2008). Les conditions de la cyclogenèse sont bien connues : des masses d'eau étendues dépassant les $26^{\circ} \mathrm{C}$ (Gray, 1968), et ce sur une profondeur de $50 \mathrm{~m}$, une instabilité atmosphérique et une force de Coriolis non nulle. Le réchauffement global, par son impact sur les températures des océans, pourrait augmenter le nombre comme l'intensité des cyclones (GIEC, 2014). Mais un lien direct entre ce réchauffement et le nombre de cyclones reste encore en débat. D'après des recherches récentes, le nombre de cyclones classés en catégorie $4 / 5$ sur l'échelle de Saffir-Simpson augmenterait de $20 \%$ à $35 \%$, même si la puissance maximale semble stable (Webster, 2005). Cette hausse de l'intensité de ce phénomène semble établie sur le bassin pacifique (Wei et Xie, 2016). En revanche, pour le bassin indien, Hoarau (2012) ne note pas d'intensification des cyclones. Mais, l'auteur ne tient compte que des cyclones de catégories 3 et supérieures et cela sur une période relativement courte (1980-2009). Un autre élément à prendre en compte semble être l'apparition d'une activité cyclonique dans des régions épargnées auparavant. En effet, les cyclones gagnent de nouvelles latitudes, $53 \mathrm{~km}$ vers le nord dans l'hémisphère nord et $63 \mathrm{~km}$ vers le sud dans l'autre hémisphère, et ce par décennie (Kossin James et al., 2014), sur une période de trente et un ans (1984-2014). 
2 Soumise à une situation anticyclonique permanente, notre zone d'étude centrée autour du golfe d'Aden connaît une aridité avérée. Il n'est donc pas surprenant que les études sur le climat et ses conséquences environnementales et sociales se soient concentrées dans cette région sur la récurrence de la sécheresse (Ayan et Ozer, 2014), la pénibilité des températures (Nour Ayeh et Ali Sougueh, 2017), les inondations 㹂 hors cyclones 監 (Omar Ali, 2018), et aucunement sur le risque cyclonique. Et pourtant, une série de cyclones est observée depuis 2015, prenant de court les populations. Leur émergence constitue donc un paradoxe en climatologie. Cet article se propose de comprendre cette apparente nouvelle situation climatique et ses conséquences. Il s'organise en deux parties. La première, en analysant les données sur quarante ans, essaie d'établir si des tendances dans la fréquence des cyclones se dégagent, et elle explore les mécanismes climatiques (en lien avec la cyclogenèse) de la mer d'Arabie dont le golfe d'Aden n'est qu'une petite excroissance. La seconde partie, en s'appuyant sur le dernier cyclone qui a touché Djibouti (Sagar en mai 2018), s'attache à évaluer la catastrophe découlant de ce phénomène météorologique dans une région peu préparée à la gérer.

\section{États des lieux : Vers une recrudescence des épisodes cycloniques?}

3 Pour cerner l'activité cyclonique dans la région bordant le golfe d'Aden, nous avons exploité les données du Regional Specialized Meteorological Center (RSMC) et du Tropical Cyclone Warming Center (JTWC) pour comptabiliser l'ensemble des cyclones ou tempêtes tropicales ayant touché le sud de l'Arabie et la Corne de l'Afrique entre 1979 et 2018. Le but est de répondre à deux questions. Quelle est la fréquence des épisodes cycloniques sur cette période dans la région? Quels sont les mois de forte activité?

\section{Analyse des situations cycloniques de 1979 à 2018}

4 La région faisant l'objet de cet article relève du bassin de l'océan Indien, appelé Nord Indian Ocean (NIO), et particulièrement dans sa partie ouest nommé bassin d'Arabie. Ce n'est pas à proprement parler un sous-bassin très affecté par les cyclones (illustration 1).

5 Nous y avons recensé trente phénomènes cycloniques ou pré cycloniques sur les quarante dernières années (illustration 2). Leur répartition n'est pas uniforme, car, comme tout phénomène météorologique, la formation d'un cyclone suit une logique chaotique (dans le sens «non prévisible»). Néanmoins, la rareté des cyclones dans le golfe d'Aden avant l'an 2000 contraste avec une cyclogenèse plus active depuis. Durant les vingt premières années de notre étude (1979-1998), le bassin a connu en moyenne 0,65 activité cyclonique par an, ce taux montant ensuite à 0,85 sur la période actuelle (1999 et 2018). 
Illustration 1 - Les zones à cyclones dans le monde

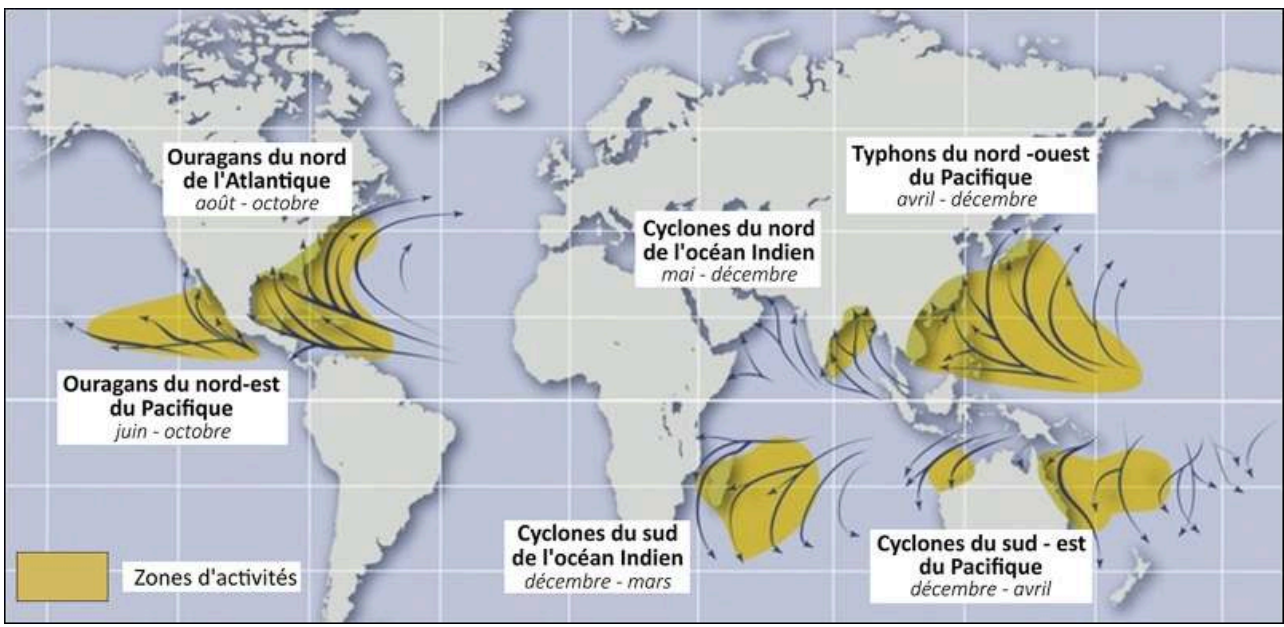

Sources : http://www.ouragans.com/; http://www.cyclonextreme.com/cyclonedicotrajectoire.htm. Auteur: J-P Cherel.

6 Cette recrudescence corrobore l'étude d'Evan et Camargo (2011) qui établissent « qu'il y a une apparente augmentation des jours de tempête cyclonique sur la période de 1992-2008 comparée à la période de 1979 à $1991 »$. Cette tendance n'est pas contredite sur le très long terme par le modèle construit par Hussain ${ }^{1}$ sur le même bassin et sur 120 ans. Néanmoins, cette évolution doit être relativisée dans la mesure où un relevé précis n'a pu se faire qu'avec le suivi satellitaire à partir des années 1960 . Ne connaissant pas les phénomènes météo d'avant 1979, il serait donc imprudent de tirer des conclusions définitives dans le cadre de cette étude.

7 Si ces phénomènes paraissent de plus en plus nombreux, ils semblent aussi de plus en plus puissants. Ainsi, toutes les dépressions tropicales ayant évolué en " cyclone » (soit huit cas sur les trente que nous avons identifiés) se sont produites depuis 1994. De plus, les cyclones dépassant la catégorie 1 n'apparaissent que depuis 2007. Chapala (2015) et Mekunu (2018) qui ont atteint la catégorie 3 sont parmi les plus puissants cyclones jamais observés dans la région. Fort heureusement, à l'approche des continents, la majorité rétrograde en tempête tropicale (illustration 3). Cette tendance doit être confirmée en étudiant les activités antérieures à notre période de référence. 
Illustration 2 - Nombre de phénomènes cycloniques sur les côtes de l'Arabie et de la Corne de l'Afrique entre 1979 et 2018

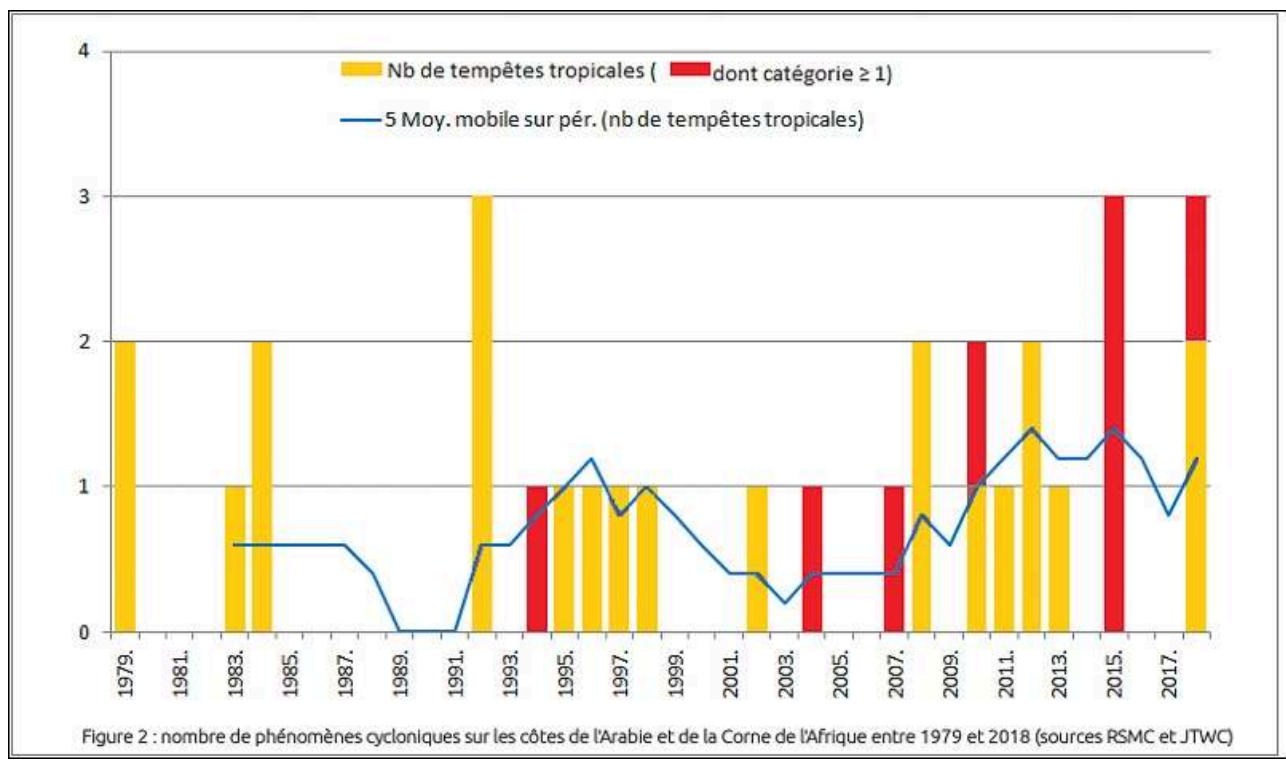

Sources: RSMC et JTWC.

8 La répartition des aires affectées montre que deux pays sont fréquemment menacés : le Sultanat d'Oman (un des rares pays ayant un système de surveillance), touché quinze fois sur notre période d'étude, et le Yémen, onze fois. Il est plus exceptionnel que les tempêtes tropicales s'enfoncent profondément dans le golfe d'Aden (deux cas seulement). Dans ce cas, malheureusement, la Somalie (pays déstructuré) enregistre autant de victimes (546) que le Yémen (578) et bien davantage que l'Oman (118). 
Illustration 3 - Les phénomènes cycloniques récents dans le golfe d'Aden (2015-2018)

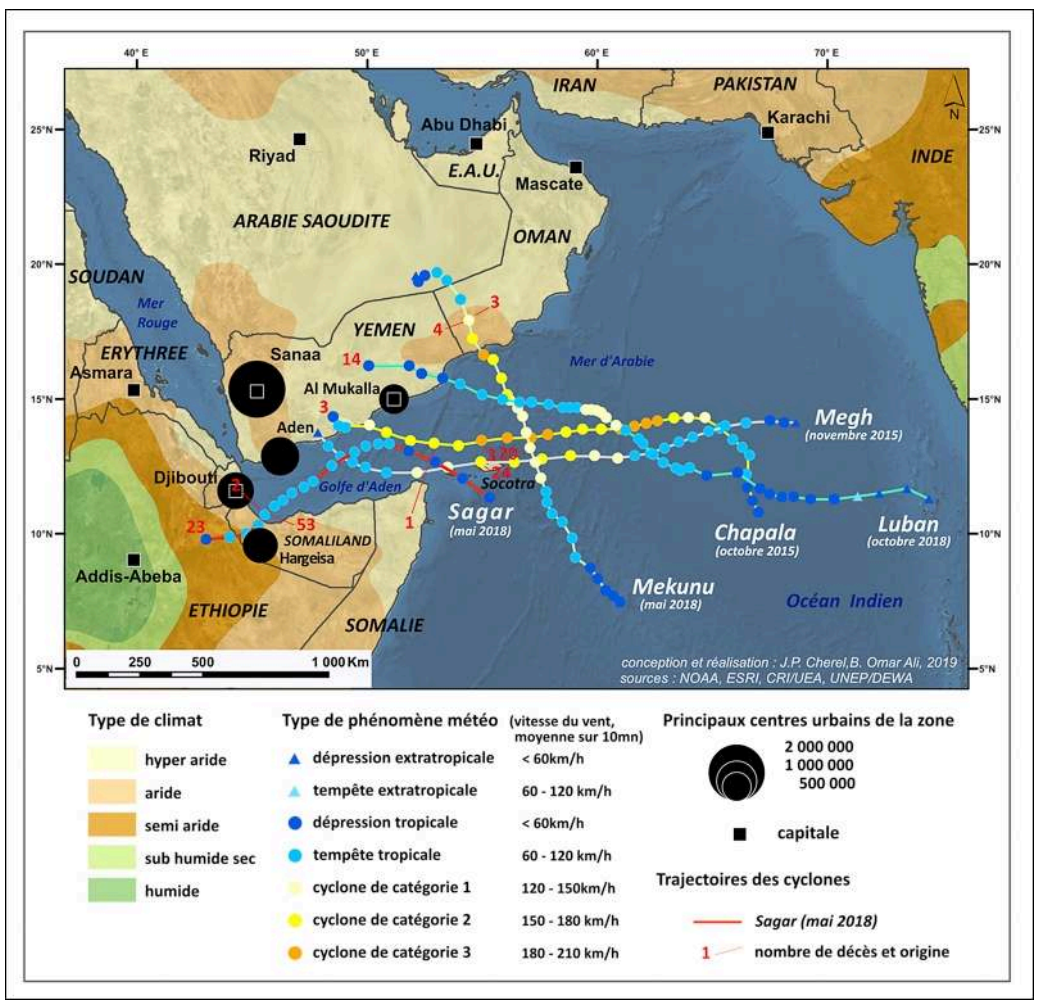

Sources : NOAA, ESRI, CRI/UEA, UNEP/DEWA ; Auteurs : J. P. Cherel et Omar Ali, 2019.

\section{Stabilité des mois touchés par les phénomènes cycloniques}

Ces dépressions ou ces cyclones se manifestent à des périodes précises de l'année. Les trente phénomènes observés se sont ainsi formés durant les deux périodes de l'année qui encadrent la mousson indienne. Ainsi, mai et juin concentrent $39,4 \%$ des cas, octobre-novembre, $40,4 \%$. Seuls $6 \%$ des cas sont recensés en juillet-août, la période la plus chaude pourtant dans la région.

10 Cette concentration de l'activité cyclonique sur deux périodes de l'année est typique de cette région. Elle est largement confirmée par d'autres études. Considérant les cyclones sur trente ans (1979-2008), Evan et Ramanathan (2011) notent la distribution bimodale des jours de tempêtes cycloniques sur la mer d'Arabie, «avec un pic d'activité survenant en mai-juin et un autre en octobre-décembre, avec une décroissance au plus fort de la mousson indienne ». Sur le très long terme (120 ans), ce caractère bimodal ne se dément pas, Hussain et al. (2011) révélant ce même type de répartition bimodale (Tableau 1). Ce comportement est singulier, dans la mesure où pour les autres bassins de l'hémisphère nord "le maximum d'occurrence se situe entre la fin de l'été et le début de l'automne boréal $»^{2}$ lorsque la chaleur accumulée est au maximum.

Tableau 1 - Répartition des cyclones de 1891 à 2015, sur le bassin de la mer d'Arabie

\begin{tabular}{|l|l|l|l|l|l|l|l|l|l|l|l|}
\hline Janv & Fév & Mars & Avril & Mai & Juin & Juillet & Août & Oct & Nov & Déc. & total \\
\hline
\end{tabular}




\begin{tabular}{|l|l|l|l|l|l|l|l|l|l|l|l|}
\hline 1 & 0 & 0 & 8 & 33 & 45 & 9 & 2 & 43 & 37 & 9 & 194 \\
\hline
\end{tabular}

Sources : PMD, Hussain et al., 2011.

11 La distribution mensuelle des cyclones demeure donc ici remarquablement stable, même si, pour notre période d'étude, il est possible de déceler une fréquence légèrement accrue de ces phénomènes ainsi qu'au renforcement de leur intensité. Les trajectoires conservent une direction sud-est et nord-est, pour le bassin NIO (quoique pour les derniers cyclones, la direction est souvent est-ouest) conforme à la direction du flux d'alizé dominant dans l'hémisphère nord. Pourtant, beaucoup de questions demeurent. Pourquoi cette distribution bimodale alors que dans le reste du monde, elle est largement unimodale ? Pourquoi les mois les plus chauds restent-ils les plus stables climatiquement sur le bassin de la mer d'Arabie? A-t-on un début d'explication à la recrudescence de ces phénomènes?

\section{Cyclogenèse et facteurs de stabilité et d'instabilité sur le golfe d'Aden}

12 Le golfe d'Aden et la mer Rouge sont parmi les mers les plus chaudes du monde, les terres qui les entourent étant connues quant à elles pour avoir des températures très élevées particulièrement en été, comme le montre le cas de Djibouti sur ces mois (Tableau 2). Ces mers satisfont donc en apparence à la première condition pour activer un cyclone, celle de la présence d'eaux très chaudes, de l'ordre de $26^{\circ} \mathrm{C}$, sur au moins $50 \mathrm{~m}$ de profondeur. Ces deux bras de mer ne sont cependant pas suffisamment étendus pour alimenter suffisamment un fort cyclone. La présence d'importantes masses continentales ne permet pas généralement aux dépressions tropicales formées sur le bassin de prendre de l'ampleur et une fois nées, elles n'ont donc pas beaucoup de temps pour se développer (Ashobaa, par exemple, s'est désintégrée en 2015 au large de l'Oman).

Tableau 2 - Températures de la ville de Djibouti (1960-2017)

\begin{tabular}{|l|l|l|l|l|}
\hline & $\begin{array}{l}\text { Température } \\
\text { moyenne } \\
\text { annuelle }\left(^{\circ} \mathrm{C}\right)\end{array}$ & $\begin{array}{l}\text { Moyenne des } \\
\text { températures } \\
\text { minimales }\left(^{\circ} \mathrm{C}\right)\end{array}$ & $\begin{array}{l}\text { Moyenne des } \\
\text { températures } \\
\text { maximales }\left({ }^{\circ} \mathrm{C}\right)\end{array}$ & $\begin{array}{l}\text { moyennes des } \\
\text { températures max du } \\
\text { mois le plus chaud, } \\
\text { juillet }\left({ }^{\circ} \mathrm{C}\right)\end{array}$ \\
\hline $\begin{array}{l}\text { Station } \\
\text { aérodrome }\end{array}$ & 30,1 & 22,97 & 37,20 & 45,34 \\
\hline
\end{tabular}

Source : Agence nationale de la météorologie Djibouti.

13 Les cinq phénomènes cycloniques, qui ont affecté la région depuis 2015, parcourent entre 1000 et $2400 \mathrm{~km}$ sur l'océan avant de toucher terre (Tableau 3). Sagar, s'il a parcouru près de $1000 \mathrm{~km}$, s'est formé à environ $300 \mathrm{~km}$ des côtes somaliennes. C'est le fait de se glisser dans le golfe d'Aden qui lui a permis de rallonger sa survie en mer. En comparaison, la distance moyenne dans le bassin atlantique est de l'ordre de $3000 \mathrm{~km}$ à 3500 km sur le Pacifique ouest ${ }^{3}$. 
Tableau 3 - Distance parcourue par les phénomènes cycloniques 2015-2018

\begin{tabular}{|l|l|}
\hline cyclone & Distance parcourue en mer $(\mathrm{km})$ \\
\hline Sagar & 1000 \\
\hline Mekunu & 1200 \\
\hline Chapala & 1800 \\
\hline Megh & 2300 \\
\hline Luban & 2400 \\
\hline moyenne & 1740 \\
\hline
\end{tabular}

Distances établies d'après les trajectoires données à la figure 3.

À l'image du bassin caraïbe ou de l'Asie de l'Est, la façade orientale de la Corne de l'Afrique est théoriquement sujette aux cyclones. Obock, à $12^{\circ} \mathrm{N}$ de latitude en République de Djibouti, est par exemple à la latitude de la Martinique. Seul le sud de la Somalie est protégé en raison de sa proximité avec l'Équateur. Durant l'été, notre région fait partie de la grande Zone de Convergence InterTropicale (ZCIT). L'instabilité atmosphérique régnant dans la ZCIT est un facteur fondamental pour le déclenchement d'une situation cyclonique puisqu'elle permet une forte ascendance de l'air. Mais comme nous l'avons noté, tous les cyclones ayant affecté la région évitent cette saison. Il existe donc bien une singularité de cette région qui échappe au schéma saisonnier.

Au-delà de l'étroitesse du sous-bassin de la mer d'Arabie, cette singularité doit s'expliquer par la dynamique générale de la circulation atmosphérique, dynamique qui serait responsable d'une certaine stabilité des masses d'air. L'arrivée de la ZCIT met en place un flux de sud-ouest, appelé mousson. Mais la disposition du relief joue, dans la Corne du moins, contre la propagation de cette mousson. En effet, ces masses d'air sont freinées par les hautes terres éthiopiennes (qui les délestent de leur humidité, l'effet orographique s'ajoutant à l'effet de convergence). Dans la partie nord de la Corne de l'Afrique, les précipitations sont donc d'été (Nicholson, 2013). Pourtant à l'échelle de Djibouti, le régime pluviométrique ne présente pas ce profil d'été commun pour la région, les rares précipitations se produisant en saison fraîche (octobre-mars). D'un autre côté, même s'il y a une petite activité cyclonique, cette station ne montre pas clairement une instabilité plus grande sur le long terme. Les données dont nous disposons, sur plus d'un siècle pour la station de Djibouti (illustration 4), montrent même un affaiblissement des rares précipitations sur les vingt dernières années. Et les minimums absolus se concentrent sur ces mêmes dernières années. Ces données vont dans le sens des sécheresses récurrentes dans la région $(2011,2015,2017,2019)$ alors même que la cyclogenèse paraît un peu plus active. Nous pourrions donc juste avancer que le climat local est devenu plus imprévisible, du moins sur les vingt dernières années. 
Illustration 4 - Précipitations, station de Djibouti, 1901-2018

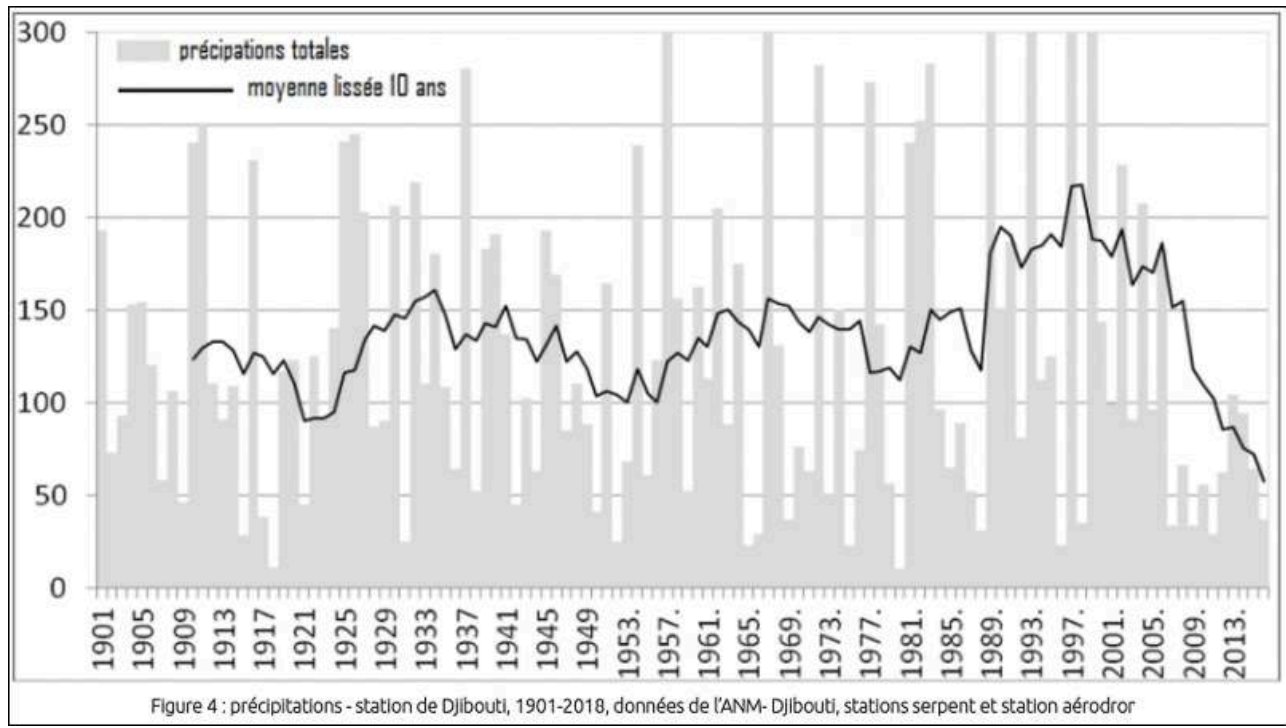

Source : données de l'ANM- Djibouti, stations Serpent et station Aérodrome.

16 La circulation d'ouest, qui s'établit durant la période de la ZCIT, crée aussi des vents continentaux qui ont tendance à s'opposer à la venue d'un cyclone sur le golfe d'Aden, même s'il se forme sur le bassin nord de l'océan Indien. Lorsque cette "mousson se déclenche, un fort cisaillement vertical se développe à travers la mer d'Arabie, empêchant la formation d'un cyclone tropical durant juillet et août " (Evan et Ramanathan, 2011). Cet important cisaillement est noté durant ces mois par plusieurs auteurs (Gray, 1986 ; Evan et Camargo, 2011).

Ainsi donc, ce n'est que lorsque le flux d'est se rétablit, avec la fin de la mousson indienne, qu'un éventuel cyclone peut prendre le chemin du golfe d'Aden. D'un autre côté, la fin de la mousson indienne impacte négativement le courant de Somalie et donc le refroidissement des eaux marines par upwelling, tout en réduisant le cisaillement vertical de l'air. Ce contexte, favorable aux cyclones dans le golfe d'Aden est assez court, se matérialisant juste avant la mise en place de la mousson indienne (mai-juin) et avant l'hiver boréal (octobre-novembre), lorsque la mer est encore assez chaude. Ces deux fenêtres d'opportunité expliquent le caractère bimodal de la répartition des cyclones sur notre bassin.

\section{Quelles causes de l'activité cyclonique dans le golfe d'Aden ?}

Malgré les obstacles à l'incursion des cyclones dans la région, les données collectées montrent qu'une activité cyclonique n'est pas à exclure. Le réchauffement global est souvent accusé d'accroître la cyclogenèse à cause de l'augmentation de la température de l'océan, condition préalable au déclenchement d'une dépression, puis d'un cyclone. Qu'en est-il sur le sous-bassin de la mer d'Arabie?

Pour valider l'hypothèse d'un réchauffement de l'océan, il faut connaître la température de la mer d'Arabie et son évolution. Une première indication est la corrélation faite par Maneesha et Manasa (2015). Sur la période 1990-2013, excluant les cyclones ayant touché la région récemment (2015-2018), ces auteurs notent bien une augmentation de la température de surface (SST pour Sea Surface Temperature) 
parallèlement à l'augmentation de la fréquence des cyclones en mer d'Arabie. Cependant cette augmentation n'est pas jugée significative. Leur étude montre, en outre, une croissance corrélative entre la température de surface et l'indice de dissipation d'énergie indiquant la force d'un cyclone (PDI). Pourtant, selon les mêmes auteurs, l'augmentation de la SST semble concerner la période pré mousson alors que l'intensité des cyclones se renforce après la fin de la mousson indienne. Ces analyses montrent une plus faible corrélation entre le SST et le $\mathrm{PDI}^{4}$ (indicateur de la force d'un cyclone) dans la période pré-mousson (mai-juin) que post-mousson (octobrenovembre), surtout pour le bassin d'Arabie, alors que la fréquence des cyclones est plus importante durant la période post-mousson. Les auteurs s'accordent toutefois sur une chose, c'est que «la SST seule n'est pas le paramètre déterminant la variabilité climatique des cyclones dans la région NIO » (Maneesha et Manasa, 2015). Ils pointent donc un mécanisme de la cyclogenèse très complexe qui ne peut être simplement réduit à la température de surface de l'eau. Mais là aussi il faudrait être prudent, leurs analyses ne concernant qu'une très courte période.

Evan et Ramanathan (2011) suggèrent d'autre part que «l'augmentation de l'intensité des cyclones arrivant à terre est la conséquence des émissions régionales d'aérosols polluants» provenant principalement du sous-continent indien. Cette pollution de l'atmosphère au-dessus de la mer d'Arabie réduirait fortement le cisaillement vertical des vents (élément inhibiteur des dépressions et cyclones tropicaux), cisaillement important durant la période de ZCIT. Pourtant, par la réduction du rayonnement au sol qu'elle induit, cette pollution issue du sous-continent indien se traduirait par une baisse de la température de surface, ce qui est contradictoire avec les conclusions de la précédente étude.

21 Ces premières indications pointent seulement le fait que le déclenchement d'un cyclone en mer d'Arabie est loin d'être mono factoriel. Certes, une SST importante (plus de $26^{\circ} \mathrm{C}$ ) est une condition nécessaire, mais loin d'être suffisante. La circulation atmosphérique lors de la période de mousson, le courant de Somalie qui en découle et même la pollution anthropique issue du sous-continent indien jouent d'une manière ou d'une autre sur la cyclogenèse. Il est encore plus délicat de relier l'activité cyclonique importante dans la région depuis trois ans à une simple température de surface de la mer (SST). On entre là dans les limites d'interprétation liées à la variabilité des phénomènes extrêmes.

\section{Impacts socio-économiques des phénomènes cycloniques : l'exemple des effets de Sagar à Djibouti}

22 La République de Djibouti, au fond du golfe d'Aden, est relativement peu touchée par les cyclones. Sagar (mai 2018) est le premier phénomène cyclonique subi par ce pays depuis la tempête tropicale 1A de 1984, époque où la ville n'avait que 150000 habitants. Cette partie interroge donc les effets de ce cyclone dans le cas spécifique de la ville Djibouti, ville peu préparée à gérer des phénomènes météorologiques de cette ampleur.

\section{Le cyclone de Sagar}

23 Le système cyclonique s'est formé dans le golfe d'Aden à partir du 16 mai. Le 18, il atteint le stade de tempête tropicale (vents jusqu'à $120 \mathrm{~km} / \mathrm{h}$ ) et prend le nom de Sagar. 
Il touche les côtes du Somaliland le 19 pour balayer Djibouti entre le 19 et le 20 mai (illustration 5$)$. Au-delà des vents $(70 \mathrm{~km} / \mathrm{h}$ sur Djibouti), ce sont les précipitations intenses qui ont causé le maximum de dégâts. L'équivalent d'une année moyenne de précipitations $(110 \mathrm{~mm})$ s'abat en effet en moins de $24 \mathrm{~h}$, générant d'importantes inondations et la crue éclair de l'oued Ambouli qui traverse Djibouti-ville.

Illustration 5a - Cyclone Sagar sur la Corne de l'Afrique le 19 mai 2018 (9 h)

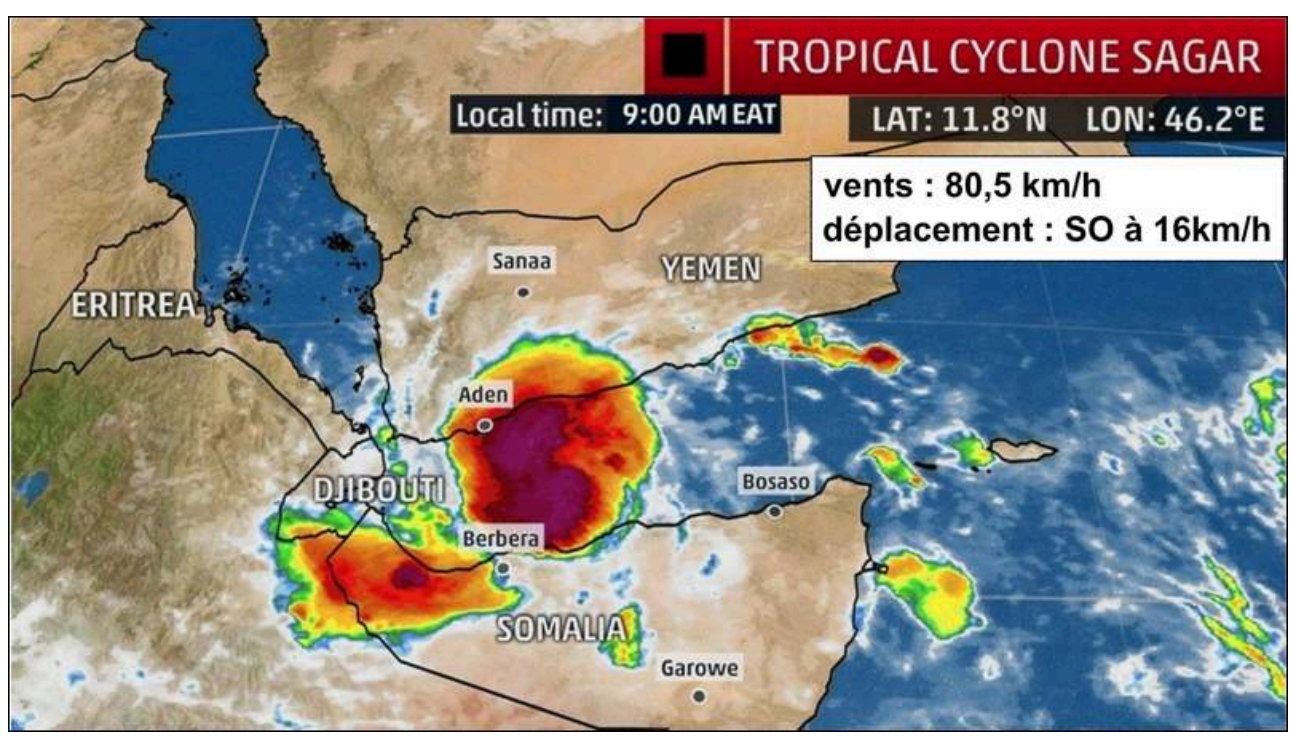

Source : https://weather.com/storms/hurricane/news/2018-05-17-tropical-cyclone-sagar-gulf-ofaden-yemen-somalia-djibouti

Illustration 5b - Cyclone Sagar sur la Corne de l'Afrique le 19 mai 2018 (17 h)

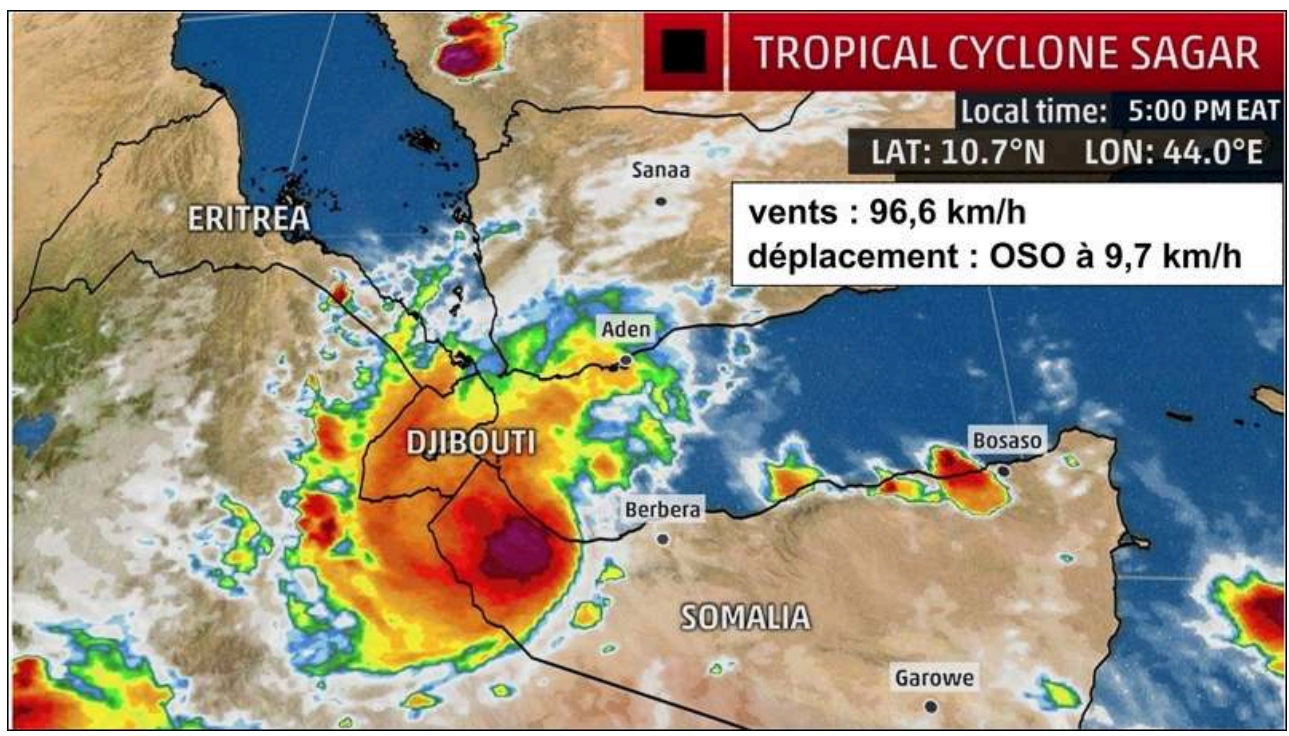

Source : https://weather.com/storms/hurricane/news/2018-05-17-tropical-cyclone-sagar-gulf-ofaden-yemen-somalia-djibouti 


\section{Recueil et analyse des données relatives au cyclone Sagar} d'enquêtes sur le terrain), nous avons pu également utiliser des données cartographiques UNITAR-UNOSAT (Operational Satellite Application Programme), pour procéder à une meilleure analyse spatiale de la catastrophe. La principale carte produite par cet organisme propose une typologie des dommages en comparant des images satellites prises avant et après le passage du cyclone. À partir de ces images à très haute résolution (de l'ordre de $50 \mathrm{~cm}$ ), trois secteurs ont été délimités en fonction de l'intensité de l'inondation (Tableau 4). La cartographie de ces secteurs récupérée sous la forme de fichiers vectoriels nous a permis d'estimer les surfaces correspondant à chacun de ces types.

Tableau 4 - Zones inondées et types de dommage

\begin{tabular}{|c|c|c|c|}
\hline $\begin{array}{l}\text { Différents secteurs } \\
\text { inondés }\end{array}$ & Secteurs à dommages possibles & $\begin{array}{l}\text { Secteurs à } \\
\text { dommages modérés }\end{array}$ & $\begin{array}{l}\text { Secteurs à dommages } \\
\text { importants }\end{array}$ \\
\hline Type de dommages & $\begin{array}{l}\text { Pas de dommages visibles sur } \\
\text { les images satellites }\end{array}$ & Dommages partiels & $\begin{array}{l}80 \% \text { de ce secteur } \\
\text { inondés }\end{array}$ \\
\hline $\begin{array}{l}\text { Superficie de la } \\
\text { zone }\end{array}$ & $0,6 \mathrm{~km}^{2}$ & $14,4 \mathrm{~km}^{2}$ & $25,1 \mathrm{~km}^{2}$ \\
\hline
\end{tabular}

Source : UNITAR, consulté le 20 mai 2018. https://unitar.org/maps/map/2810

27 Une première lecture nous donne l'ampleur des inondations dues à Sagar. En effet, sur les $40,1 \mathrm{~km}^{2}$ des deux communes, $25,1 \mathrm{~km}^{2}$ ont été sévèrement touchés (soit $63 \%$ de ces communes), avec $80 \%$ de la surface sous les eaux (illustration 6) 


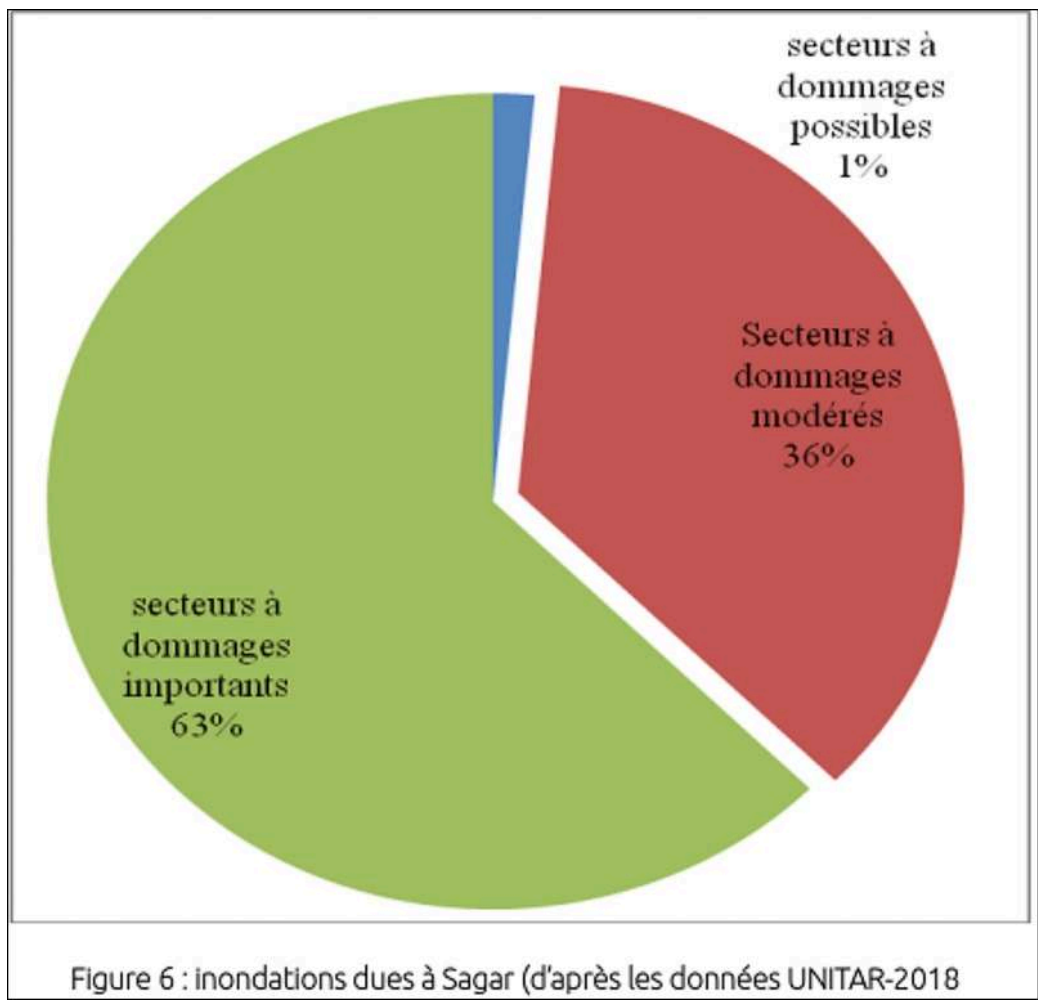

Sources : données UNITAR, 2018.

\section{Les zones exposées}

Plus encore que dans les autres villes du pays comme Arta et Tadjourah, les conséquences de ces inondations sont lourdes dans la capitale qui concentre les enjeux humains et économiques dans des zones inondables. Les quartiers les plus impactés sont ceux de la commune de Boulaos qui s'étend dans la partie la plus exposée aux inondations de la capitale, parce que quasiment située au niveau de la mer. L'illustration 7 met en évidence l'accroissement de l'agglomération de Djibouti. À partir de son site originel (commune de Ras Dika), la ville s'est en effet étendue vers le sud en direction de l'oued Ambouli sur des zones d'une altitude inférieure à $1 \mathrm{~m}$, avant de partir à l'assaut des hauteurs de Balbala à l'ouest. 
Illustration 7 - Extension de l'agglomération de Djibouti depuis 1953

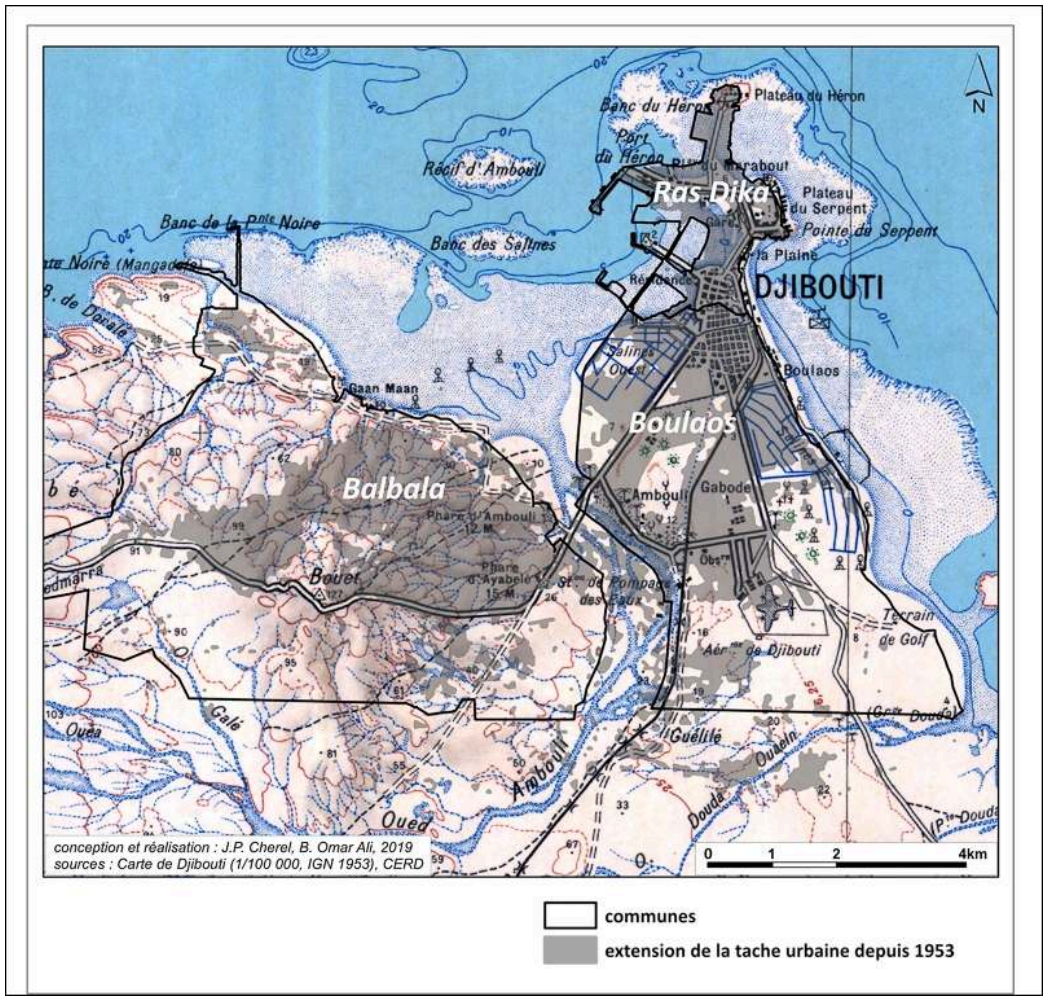

Sources : Carte de Djibouti (1/100 00, IGN, 1953, CERD. Auteurs : J.-P. Cherel et B. Omar Ali.

Les quartiers de Boulaos concentrent donc l'essentiel des enjeux lors d'une inondation. Nous avons croisé dans l'illustration 8 les chiffres de population de 2015 (estimation fournie par la DISED de Djibouti) avec la cartographie des dommages réalisée par l'UNITAR à partir de l'imagerie satellitaire (UNITAR, 2018). Elle fait clairement apparaître que les habitants des quartiers de Boulaos (plus de 224000 personnes) ont été a priori les plus touchés, beaucoup plus que dans les quartiers de Ras Dika (11 000 hab.) ou Balbala (310 000 hab.). 
Illustration 8 - Zones inondées par le cyclone Sagar le 20 mai 2018

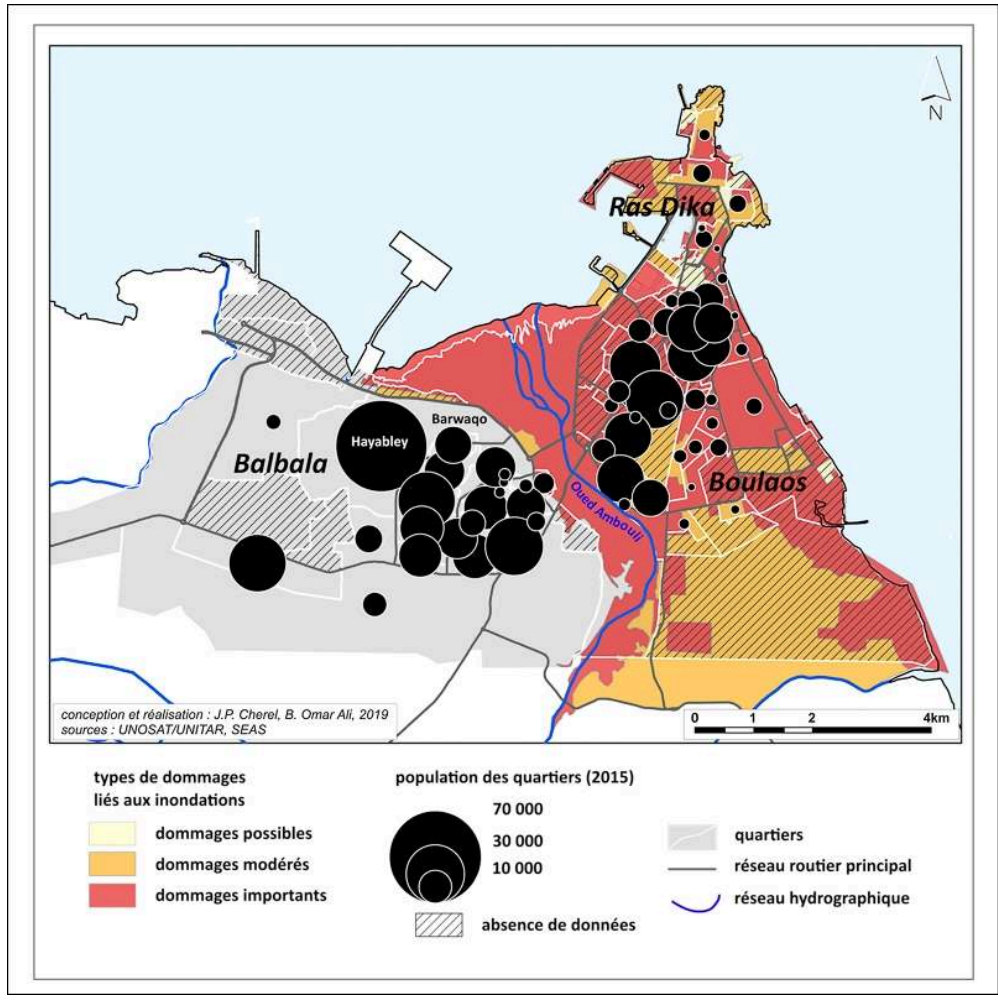

Sources : UNOSAT/UNITAR, SEAS. Auteurs : J.-P. Cherel et B. Omar Ali.

\section{Bilan humain des inondations consécutives au cyclone Sagar}

Les premières évaluations réalisées par le gouvernement djiboutien avec l'appui des agences onusiennes ont permis de recenser les dégâts humains de cette catastrophe. Le bilan humain s'élève à deux morts dans la capitale. Selon la direction de la Protection civile, il s'agit de personnes qui ont tenté de traverser l'oued Ambouli avec leurs véhicules, la route reliant le centre-ville et les quartiers de Balbala Nord (comme Barwaqo et Hayabley) n'ayant pas été fermée par les autorités de la ville.

31 Au-delà de ces pertes en vies humaines, le chiffre du bilan humain a connu une fluctuation durant les mois qui ont suivi le passage du cyclone. Les premières estimations faisaient par exemple état de 25000 personnes affectées (50 000 personnes sur l'ensemble du pays). Par la suite, les autorités djiboutiennes ont affirmé qu'environ 20000 personnes avaient été sinistrées. Cette fluctuation du nombre de personnes affectées montre toute la difficulté pour les autorités djiboutiennes, et plus particulièrement pour les institutions nationales en charge de la gestion des catastrophes naturelles, de fournir un chiffrage exact du bilan d'un événement comme le cyclone Sagar. Nous avions déjà noté ces difficultés dans le cadre des missions d'évaluation des dommages réalisées suite aux inondations du 25 mars 2013 ayant également affecté la ville de Djibouti (Omar Ali, 2018).

L'illustration 9 montre que le Quartier 7, Arhiba, Quartier 4, Djebel et Quartier Sud sont les secteurs de la ville de Djibouti les plus affectés par cette catastrophe sur le plan humain. 
Illustration 9 - Secteurs et routes affectés par les inondations provoquées par Sagar, Djibouti, le 20 mai 2018

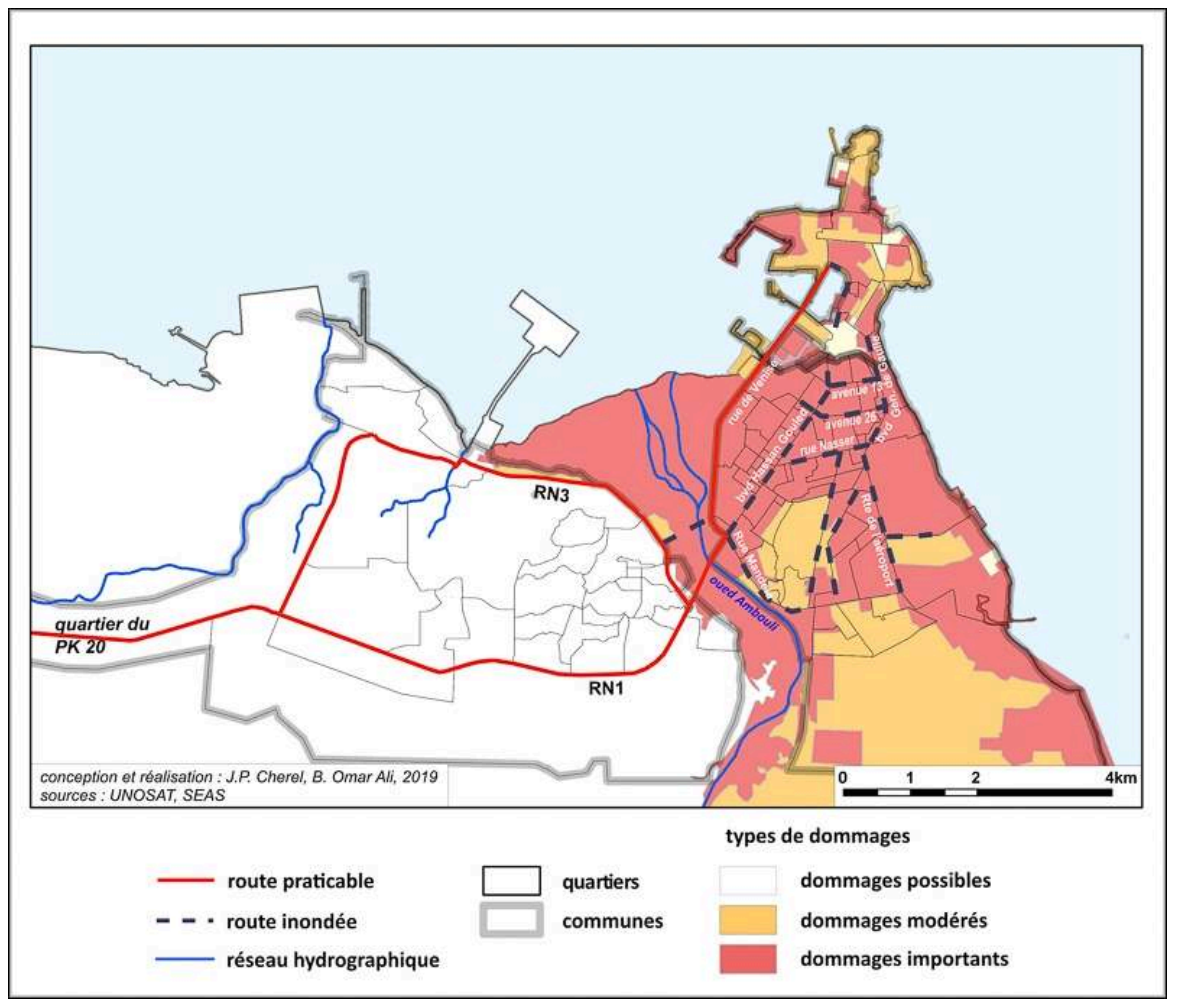

Sources : UNOSAT, SEAS. Auteurs : J.-P. Cherel et B. Omar Ali.

Afin d'évaluer l'impact humain de cette catastrophe, nous avons recensé le nombre de familles affectées dans les différents quartiers de la ville de Djibouti. D'après le résultat d'une enquête réalisée par le DISED, 1042 familles ont été sinistrées par les inondations consécutives au cyclone. Elles sont inégalement réparties entre les différents quartiers de la ville de Djibouti. Les secteurs Quartier 3, Quartier 7bis, Ambouli et Arhiba sont les quartiers de la ville regroupant le plus grand nombre de familles affectées.

Cette cartographie met en évidence la forte exposition de cette commune face aux inondations accompagnant un cyclone. Ces quartiers sont situés sur des secteurs plans de faible altitude ( 5 à $10 \mathrm{~m}$ ) en rive droite de l'oued Ambouli. « Dans certains quartiers, les habitations sont localisées dans des zones où l'occupation devait être interdite. Les autorités de la ville sont confrontées devant un fait accompli puisque les programmes d'aménagements et de planification du processus de l'urbanisation de la ville sont précédés par les installations des populations" (Omar Ali, 2018). Cette forte vulnérabilité est liée également au désengagement de l'État sur la gestion urbaine au cours des dernières décennies. Nour Ayeh (2015) notait que « le rôle de l'État est crucial dans la gestion urbaine, de la construction des quartiers, et l'entretien des infrastructures à la collecte des déchets. Son désengagement consacré par l'ajustement structurel a renforcé les forces centrifuges. Il accepte l'idée qu'il faut juste accompagner les quartiers précaires pour améliorer la survie. Cet accompagnement se traduit par la mise en place de la voirie, de l'électricité et de quelques bâtiments publics sur des crédits consentis par la Banque Mondiale, mais aussi de la France ». Les secteurs inondés correspondent bien à ceux se trouvant au niveau de la mer et proches de l'oued d'Ambouli, mais aussi habités par des populations relativement démunies. 


\section{Le bilan matériel du cyclone}

Outre les impacts humains, cette tempête a affecté les infrastructures urbaines de la ville de Djibouti comme celles des régions de l'intérieur du pays. Parmi ces infrastructures, on peut citer l'exemple du réseau routier qui a été lourdement touché par ces inondations (illustration 9). Les axes routiers reliant les différents quartiers de la commune de Boulaos ont été fermés durant plusieurs heures, voire plusieurs jours dans certains cas, bloquant ou contrariant la circulation des personnes et l'acheminement de l'aide et des secours. À chaque nouvelle inondation, on assiste au même scénario. Les grandes artères de la ville comme le boulevard Hassan Gouled (route d'Arta), le boulevard De Gaulle, les avenues 13, 26 et Nasser ont été fermées les 20 et 21 mai 2018 (illustration 10). La route qui dessert les quartiers du nord de Balbala, un des deux grands axes routiers reliant cette commune au reste de la ville de Djibouti, a été aussi fermée durant plusieurs heures. Dans Balbala même, certaines routes ont été également endommagées ce qui confirme une nouvelle fois la forte vulnérabilité de l'ensemble du réseau routier de la capitale en cas d'inondation. Cette vulnérabilité est largement due à l'insuffisance du drainage des eaux de surface, les réseaux d'évacuation des eaux pluviales et des égouts étant très mal entretenus et rapidement bouchés lors des crues. Au-delà de la capitale, le réseau routier à l'intérieur du pays a été aussi affecté par ces inondations. Un tronçon de la route nationale RN1 qui relie les régions de Tadjourah et d'Obock au reste du pays a ainsi été détruit au niveau de la localité de PK 51, ce qui a paralysé cet axe durant plus d'une journée et perturbé la circulation des personnes et des biens. À l'exception de cette portion de la RN1, la plupart des dommages sur le réseau routier sont restés concentrés sur la capitale (banque Mondiale, 2018). 
Illustration 10 - Route reliant les quartiers du nord de Balbala au reste de la ville coupée par la crue de l'oued Ambouli, le 20 mai 2018

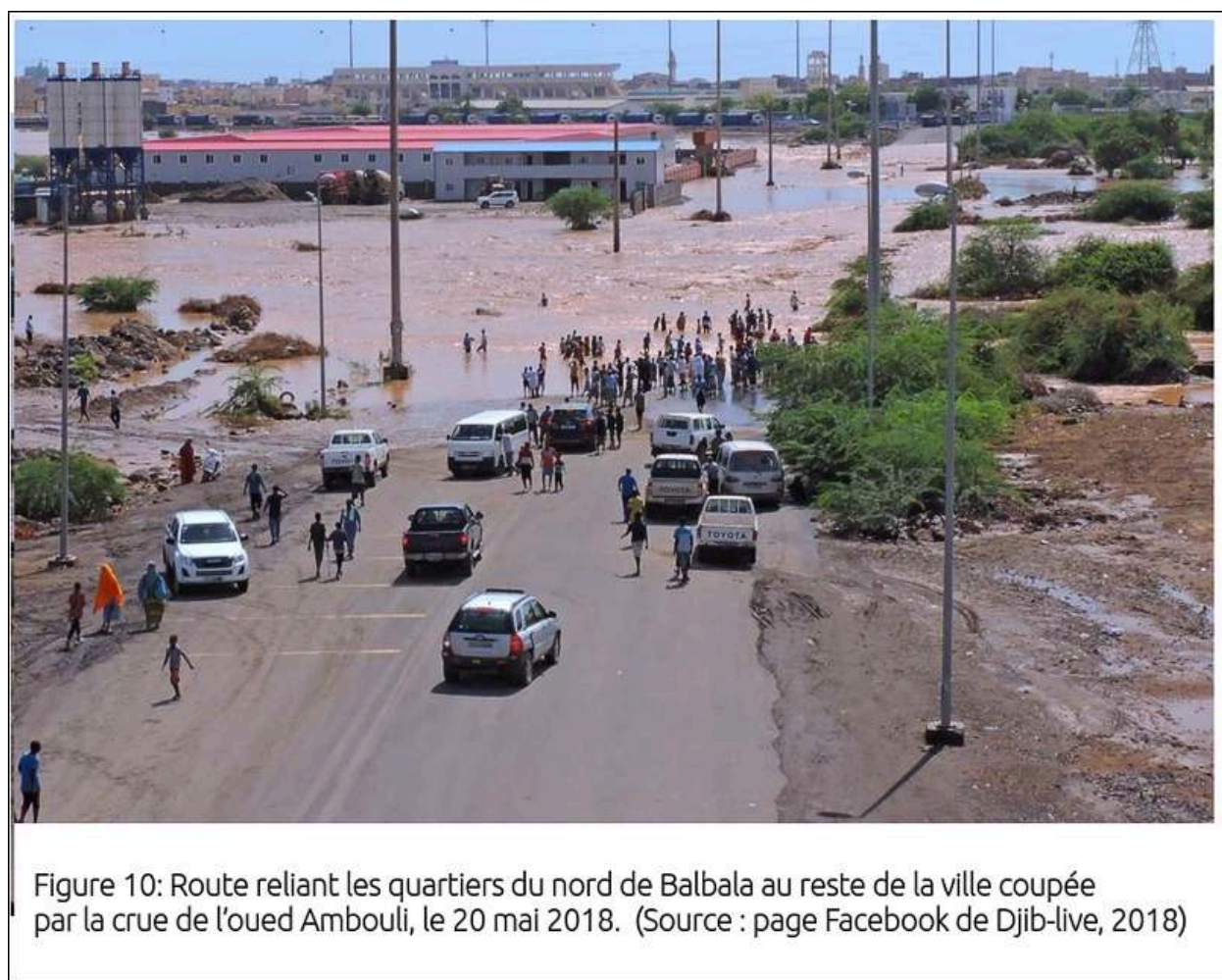

Source : Cpage Facebook de Djib-live, 2018.

Les évaluations réalisées par les agences onusiennes et les partenaires internationaux (comme la Banque Mondiale) avec l'appui du gouvernement djiboutien ont permis de quantifier les dommages consécutifs à Sagar sur les infrastructures routières. Au niveau de la ville de Djibouti, le coût des dommages est estimé à 2,2 milliards de Francs de Djibouti (FDJ) soit un total de 12 millions de Dollars (Banque Mondiale, 2018). Pour le secteur du transport, les besoins globaux de reconstruction sont estimés à 1,9 milliard FDJ (Banque Mondiale, 2018).

Outre le secteur du transport, d'autres infrastructures urbaines ont été sérieusement affectées par ce cyclone. Ce fut le cas des installations du système d'assainissement de la ville dont certaines ont été lourdement endommagées alors que d'autres ont subi des dégradations partielles. " $12 \mathrm{~km}$ de réseau ont été entièrement endommagés principalement dans les quartiers d'Einguella et Arhiba tandis que $20 \mathrm{~km}$ sont partiellement à renouveler dans d'autres quartiers" (Banque Mondiale, 2018). La réhabilitation de ces infrastructures nécessite des financements très importants. On estime qu'environ 1,6 milliard FDJ (9 millions US \$) sont nécessaires à la remise en état des infrastructures. Sagar a également entraîné la destruction des latrines de nombreux ménages de la capitale. $26,5 \%$ des ménages enquêtés par les équipes du $\mathrm{SEAS}^{7}$ ont affirmé que leurs latrines ont été complètement détruites, 58,3\% signalant des latrines moyennement endommagées. "Parmi ceux qui considèrent que leurs latrines ont été détruites, $61,8 \%$ déclarent ne pas avoir réhabilité leur latrine contre $0,6 \%$ qui ont bénéficié d'une réhabilitation à l'aide d'un organisme » (SEAS, 2018). Ces destructions sont susceptibles d'entraîner des problèmes sanitaires. Par exemple, dans 
le quartier d'Arhiba, l'eau potable a été contaminée par les eaux usées à la suite de la dégradation du réseau d'assainissement.

Le cyclone a également entraîné une aggravation de la pénurie d'eau à laquelle la ville de Djibouti se trouve confrontée, l'alimentation en eau ayant subi de graves perturbations dans les différents quartiers. Ces problèmes d'accès à l'eau s'expliquent par la destruction de deux forages qui alimentent la ville et qui sont situés au niveau de PK 20 (d'un débit de $55 \mathrm{~m}^{3} / \mathrm{h}$ ). 22,8\% des ménages des quartiers enquêtés disent avoir subi des problèmes d'accès à l'eau (SAES, 2018).

Le secteur éducatif a aussi subi des impacts importants. Certaines écoles de la capitale ont été inondées et fermées durant plusieurs jours entraînant ainsi une rupture de la scolarisation des enfants, principalement dans les quartiers de Boulaos (illustration 11). Dans les écoles affectées par les inondations, les salles de classe et le mobilier scolaire ont été lourdement endommagés. Au total, le cyclone a entraîné la fermeture de treize écoles primaires, trois collèges et un lycée technique. Dans ces différents établissements, les dégâts recensés sont la destruction des planchers et des plafonds, la dégradation des peintures, des meubles et des fournitures scolaires. La scolarité des nombreux enfants a été ainsi perturbée. "Le passage de Sagar a fait que $66,6 \%$ des ménages ont déclaré que leurs enfants n'ont pas été à l'école pendant quelques jours, mais moins d'une semaine, alors que $26 \%$ n'ont pas été du tout perturbés et que 52,5\% affirment avoir vu le matériel scolaire de leurs enfants emporté ou détruit par les eaux » (SAES, 2018). Le passage de Sagar ayant coïncidé avec la fin de l'année scolaire au niveau national, l'impact sur la scolarité des enfants a pu être atténué. Le cyclone a toutefois affecté la capitale à une période où les examens de fin d'année débutaient pour de nombreux collégiens et lycéens. Les examens de 135000 collégiens et lycéens ont été de ce fait reportés d'une semaine pour attendre la fin des opérations de nettoyage et de remises en état des établissements affectés (SAES, 2018). 
Illustration 11 - Localisation des établissements scolaires inondés par le cyclone Sagar dans les communes de Boulaos et Ras Dika, le 20 mai 2018

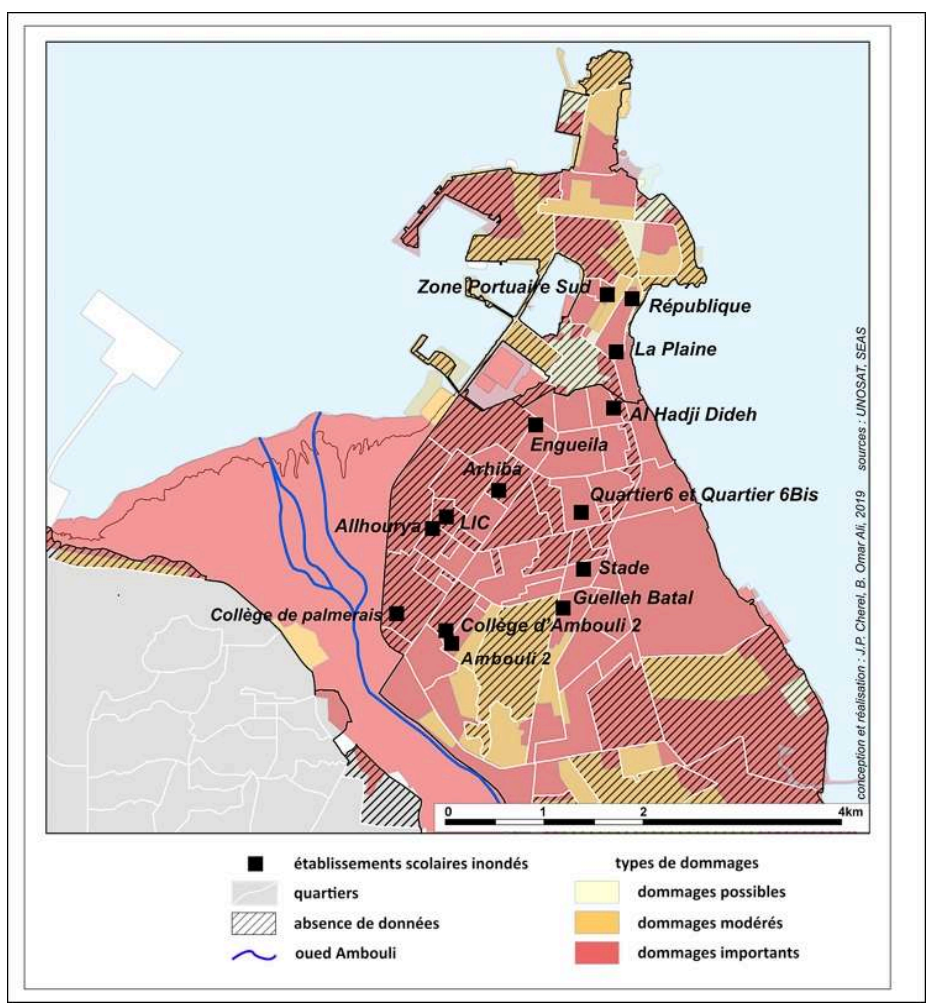

Les CDC (Centres de Développement Communautaire), infrastructures communautaires destinées aux jeunes des différents quartiers de la capitale et gérées par le secrétariat d'État à la Jeunesse et aux Sports (SEJS), ont subi également de lourds dégâts (illustration 12). Déjà fragilisés par l'absence d'entretien et le manque de ressources financières, les $C D C$ ont vu leurs toitures, leurs installations électriques et leurs mobiliers détruits par le cyclone. D'après des entretiens informels avec la directrice des CDC au niveau du SEJS, près de $50 \%$ de ces CDC ont ainsi subi des dégâts au niveau des toitures, presque $40 \%$ ayant enregistré des dommages sur les installations électriques. Ces structures communautaires furent donc fermées au public durant plusieurs semaines, alors que ce sont de véritables lieux de socialisation, de rencontres, d'échanges, de formations et de sensibilisation pour les jeunes des quartiers.

41 Au niveau de la capitale, les CDC étant d'autre part régulièrement utilisés par les autorités comme des sites refuges pouvant abriter les populations évacuées lors de graves intempéries (cas des inondations d'avril 2004 et mars 2013), leur indisponibilité suite au passage du cyclone a compliqué la tâche des autorités djiboutiennes en charge de la gestion de crise. Elle a créé en effet une situation de « sur-crise » particulièrement préjudiciable pour les sapeurs-pompiers. 
Illustration 12 - Types de dégâts causés par Sagar au niveau de CDC de Djibouti, le 20 mai 2018

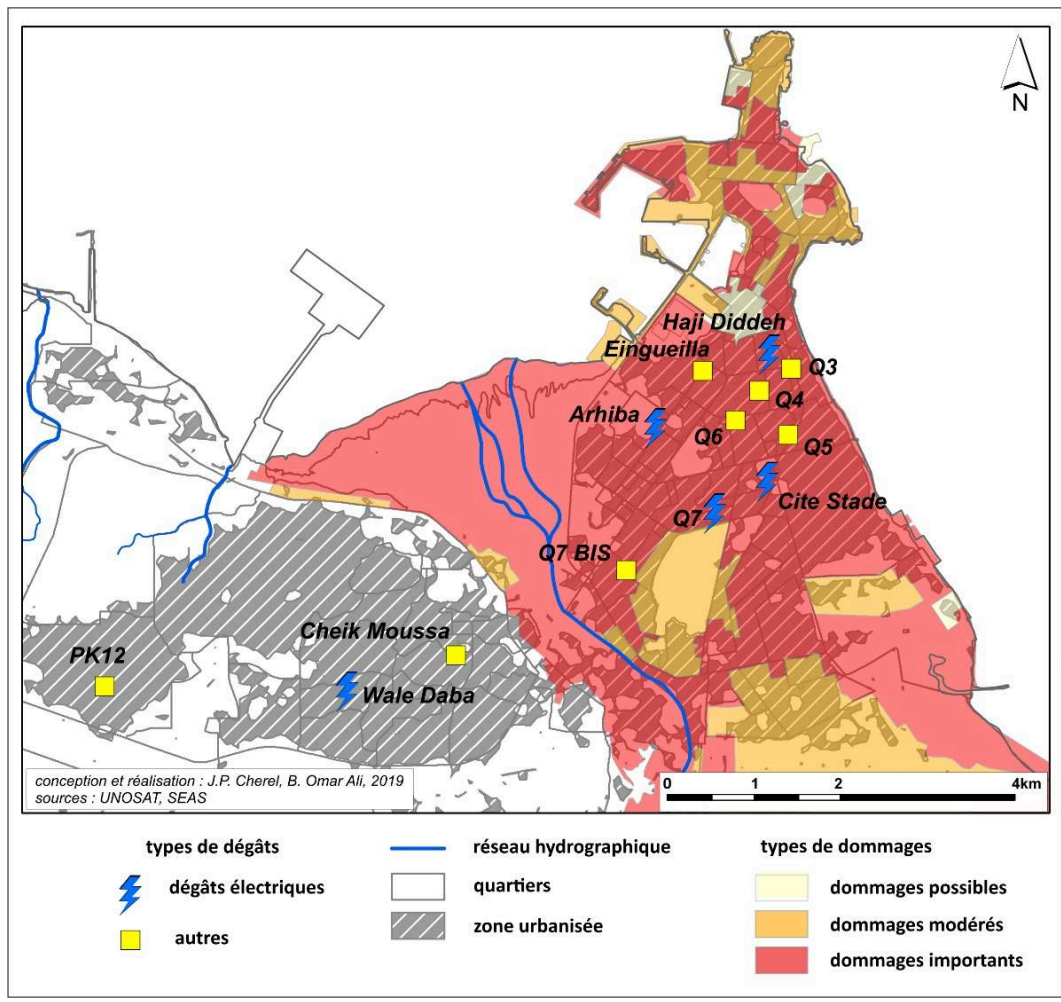

42 Selon les enquêtes réalisées par le SEAS, les pertes au niveau des biens et des matériels ont été très importantes, la valeur économique des pertes subies par les ménages atteignant près de 1,7 milliard $\mathrm{FDJ}^{8}$. Ces pertes incluent le mobilier (643 millions DJF), l'électroménager et le matériel électronique (638 millions DJF), ainsi que les moyens de locomotion (58 millions DJF) (illustration 13). 
Illustration 13 - Valeurs économiques des biens détruits par le cyclone, le 20 mai 2018, dans la ville de Djibouti (en euros)

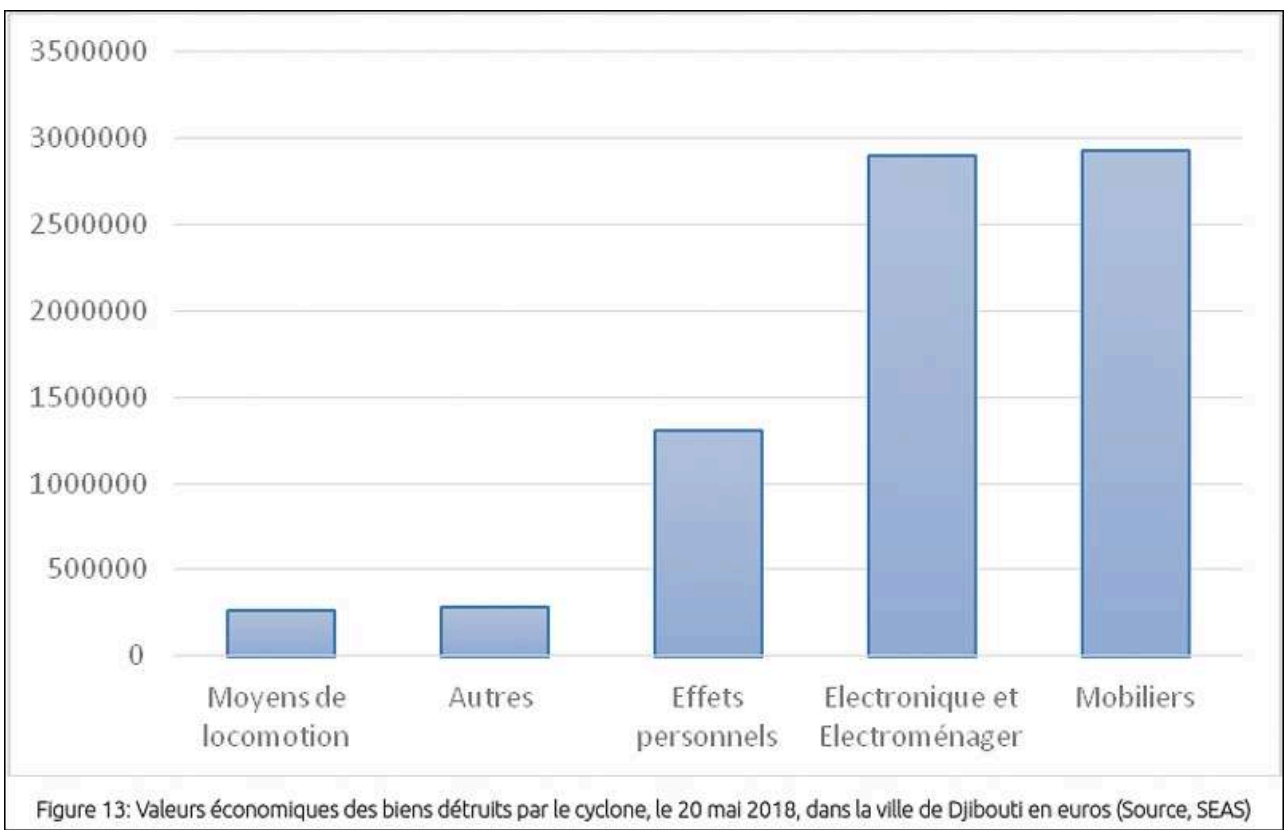

Source : SEAS

Beaucoup de ménages ont également subi des perturbations ou des coupures électriques. C'est le cas de 455 ménages sur les 1041 interrogés par la direction de la statistique. Les secteurs Arhiba, Quartier 2 et Quartiers 4 sont ceux concentrant les ménages les plus touchés (illustration 14).

Illustration 14 - Nombre de ménages ayant connu des perturbations dans l'alimentation en courant électrique, durant l'épisode Sagar, les 19 et 20 mai 2018

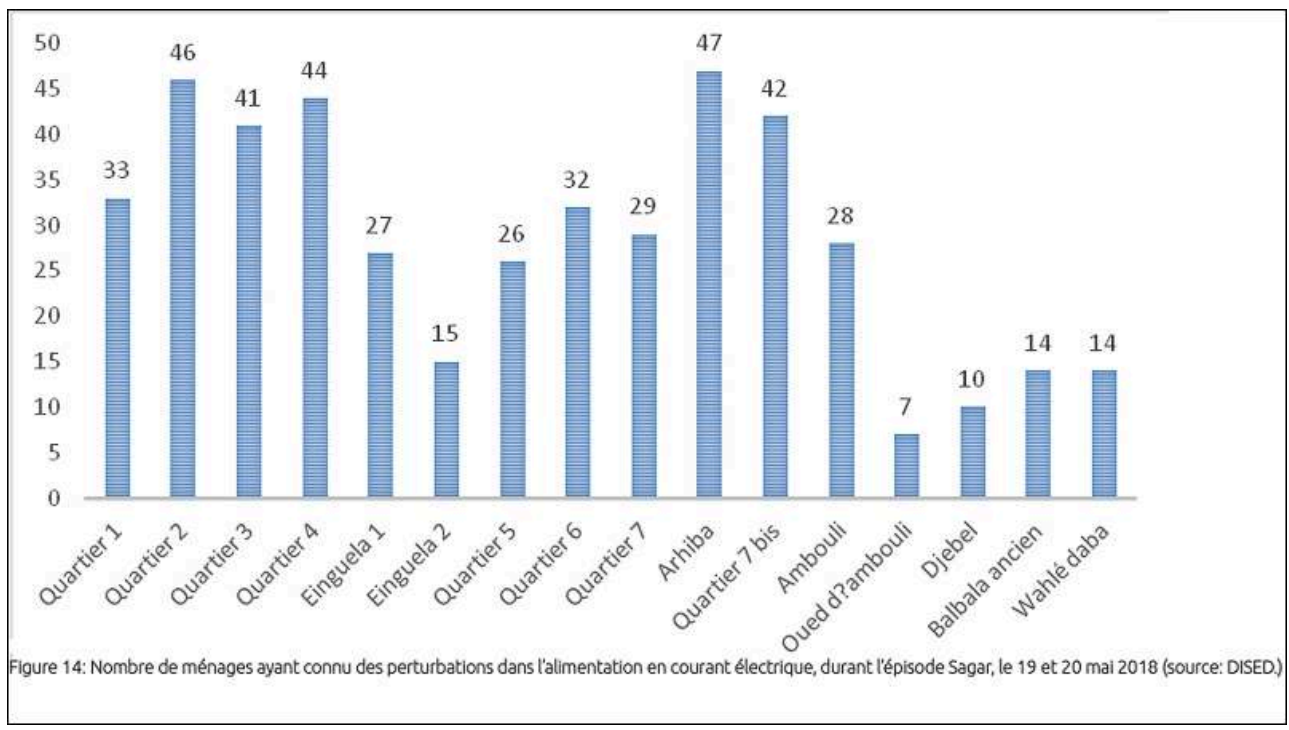

Source : DISED.

44 Ces différents chiffres témoignent de l'ampleur des pertes économiques induites par le cyclone Sagar et surtout de l'importance des biens localisés dans des zones à risque. Cette catastrophe naturelle révèle une nouvelle fois la forte vulnérabilité économique 
de la ville de Djibouti face à ce type d'aléa. Elle confirme la nécessité de mettre en place de nouvelles mesures pour mieux protéger les enjeux humains et économiques localisés dans des zones à risque, mais aussi pour renforcer leur résilience.

\section{Gestion des effets du cyclone}

Suite à cette catastrophe, le gouvernement djiboutien, appuyé par les agences onusiennes et les partenaires internationaux, a été conduit à mettre en place un ensemble de mesures et d'actions pour apporter des réponses aux dégâts causés par le passage de Sagar. Cependant, non habituées à gérer un tel évènement, les autorités djiboutiennes ont été dans un premier temps dépassées par l'ampleur de la situation. Face à l'urgence, le Premier ministre djiboutien a alors ordonné la création d'une commission ad hoc chargée de faire un état de lieux de la catastrophe. Participaient à cette commission présidée par le directeur général de la Protection civile :

- le directeur de l'Assainissement ;

- le directeur des Domaines et de la Conservation Foncière ;

- le directeur de l'Aménagement du Territoire, de l'Urbanisme et de l'Habitat ;

- le directeur de l'ADR (Agence Djiboutienne des Routes) ;

- le préfet de la ville ;

- le maire de la ville.

Cette commission avait la responsabilité du suivi de la gestion des opérations, et plus particulièrement celle de l'évacuation des eaux de pluie dans les différents quartiers de la capitale. Pour cela, une réunion de crise portant sur l'évacuation des eaux, des déchets et des boues dans les quartiers, les écoles et les CDC fut organisée avec de hauts gradés de l'armée nationale et des responsables des différentes directions du ministère de l'Intérieur. Il fut acté que les différentes forces armées participeraient aux diverses opérations envisagées (tableau 5).

Tableau 5 - Intervention des différents corps constitués dans les opérations de secours aux quartiers

\begin{tabular}{|l|l|l|l|l|}
\hline $\begin{array}{l}\text { Corps de l'armée } \\
\text { intervenant dans les secours }\end{array}$ & $\begin{array}{l}\text { Armée } \\
\text { Nationale }\end{array}$ & $\begin{array}{l}\text { Gendarmerie } \\
\text { Nationale }\end{array}$ & $\begin{array}{l}\text { Marine Nationale et } \\
\text { Garde Côte }\end{array}$ & $\begin{array}{l}\text { Police } \\
\text { Nationale }\end{array}$ \\
\hline & Quartier 6 & Quartier 1 & Quartier 3 & Arhiba \\
\cline { 2 - 6 } & Quartier 7 & Quartier 2 & Quartier 4 & Engueilla \\
\cline { 2 - 6 } Quartiers concernés & $\begin{array}{l}\text { Quartier } \\
\text { bis }\end{array}$ & $\begin{array}{l}\text { Secteur Avenue } \\
13\end{array}$ & Quartier 5 & \\
\cline { 2 - 6 } & Ambouli & $\begin{array}{l}\text { Place Mahamoud } \\
\text { Harbi }\end{array}$ & & \\
\cline { 2 - 6 } & Djebel & & & \\
\cline { 2 - 5 } & & & & \\
\end{tabular}

Source : compte rendu de la réunion de cellule de crise du 20 mai 2018 

opérations de distribution de denrées alimentaires dans les quartiers les plus touchés (notamment les quartiers 3, 7bis et Ambouli). Ces opérations ont été réalisées par des agences nationales comme le SEAS, l'ONARS', des associations ou des ONG nationales, des organismes multilatéraux (PAM, Unicef, FAO, HCR...), des organismes bilatéraux (Ambassade, USAID) et des organisations du secteur privé. Sur un total de 1041 ménages enquêtés, 495 ménages disaient avoir reçu ce type d'aide, soit 47,5\% des ménages (illustration 15). Cela montre une assez faible couverture de cette opération mise en place dans les jours qui ont suivi le passage de Sagar.

Illustration 15 - Nombre de ménages ayant reçu des aides alimentaires à la suite du cyclone Sagar en 2018

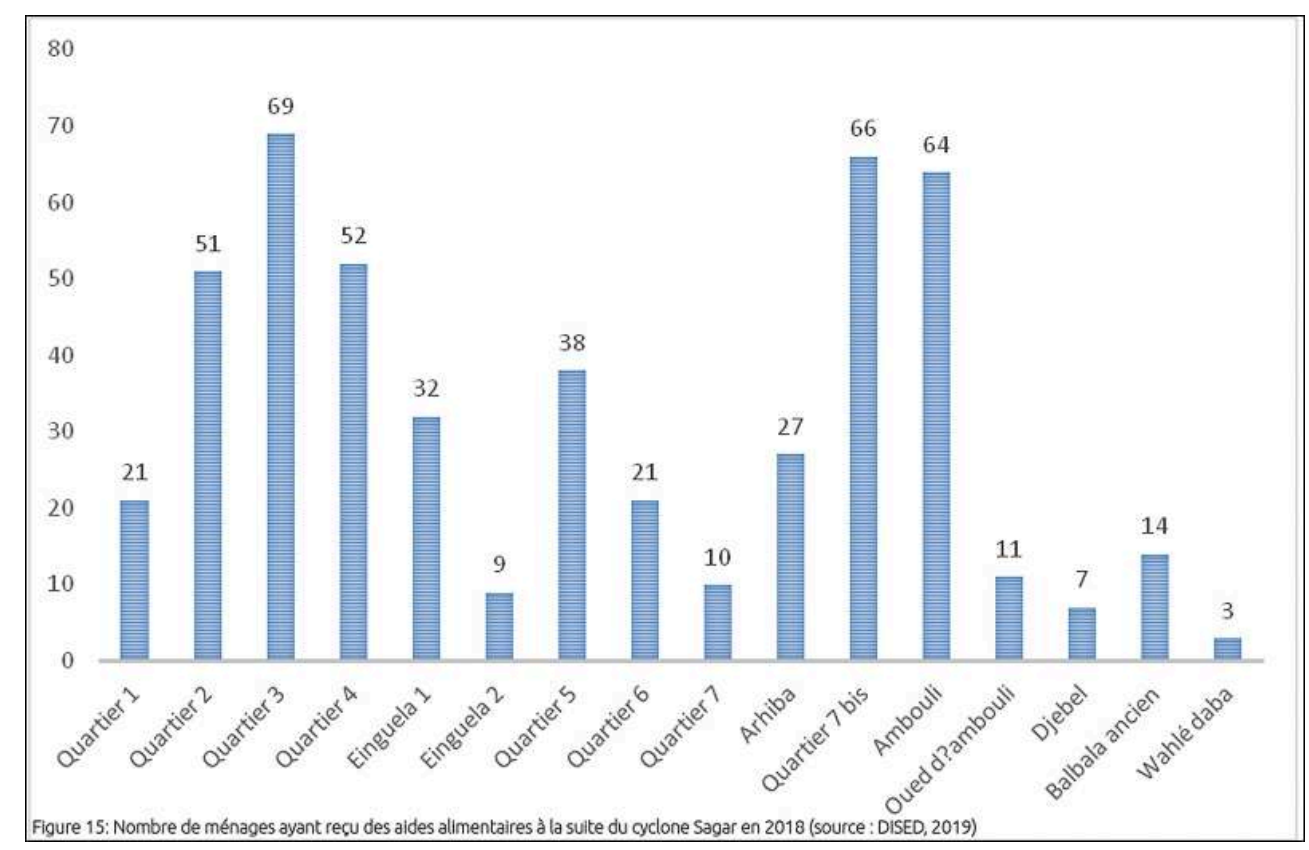

Source : DISED, 2019.

EchoGéo, 51 | 2020 
aide d'urgence a toutefois permis à de nombreux ménages de bénéficier d'une aide complétant celle de leurs proches ou amis pour surmonter une éventuelle pénurie en produits alimentaires.

\section{Conclusion}

Plusieurs éléments peuvent être tirés de cet article. Nous savions que la petitesse du bassin de la mer d'Arabie, le phénomène d'upwelling sur les côtes somaliennes, la subsidence de l'air, y compris en été, en raison de la situation d'abri des régions du golfe d'Aden, étaient autant d'éléments rendant cette région du golfe d'Aden peu sensible aux problèmes des cyclones tropicaux. Pourtant, sur les quatre dernières décennies, nous avons relevé plusieurs phénomènes cycloniques qui ont affecté ces côtes. Si Hoarau note une absence d'augmentation des cyclones de catégorie 3 et plus sur l'ensemble du bassin indien, Hussain accrédite une tendance à la hausse de l'activité cyclonique en général sur le sous-bassin d'Arabie sur une très longue période (120 ans). Notre étude semble corroborer les conclusions de ce dernier auteur, avec trente phénomènes cycloniques affectant cette région. Il est à noter que tous les cyclones de catégorie 1 et supérieurs se sont développés depuis 1994. Au vu de la courte période concernée et de la rareté de ces phénomènes, il est très délicat d'y voir une conséquence directe du réchauffement global. Il faut, pour cela, d'abord voir si la tendance que nous avons observée se confirme dans les années à venir. En outre, le mécanisme climatique en œuvre sur ce bassin est si complexe que l'impact du réchauffement global sur chaque paramètre impliqué dans la cyclogenèse est loin d'être établi. Une modélisation poussée du système prenant en compte le comportement de chacun de ces paramètres (température de surface et upwelling somali, cisaillement vertical de l'air, comportement de la mousson indienne et généralement de la ZCIT) permettrait à l'avenir de mieux appréhender le système climatique de la mer d'Arabie. Le problème majeur pour une telle modélisation reste le manque d'une connaissance très fine de chaque élément du système.

Cependant, la moindre tempête tropicale déstabilise un système socio-économique bien ancré dans le sous-développement, et ce dans la plupart des pays de la région. D'un côté, les probabilités d'un épisode cyclonique semblent être plus fortes, du moins, sur les quarante dernières années. De l'autre, les populations riveraines du golfe d'Aden (qui sont de plus en plus nombreuses) n'ont pas les outils et les moyens pour se protéger de ce phénomène et en atténuer ses conséquences. Au-delà des pays déstructurés, comme la Somalie et le Yémen qui subissent juste une calamité supplémentaire, un État qui fonctionne, comme Djibouti, voit sa capitale paralysée et plus de 200000 de ses habitants inondés par un cyclone, déjà rétrogradé en tempête tropicale lorsqu'il a touché le pays (Sagar). Les autorités, en charge de la gestion de cette catastrophe, ont été rapidement dépassées. L'épisode Sagar a ainsi crûment révélé des réalités géographiques (deux communes sur trois au niveau de la mer) et socioéconomiques (insuffisance des infrastructures, pauvreté des populations) ainsi que les faibles capacités de gestion des crises, qui sont autant d'éléments sources de la vulnérabilité de cette capitale. Devant cette apparente nouvelle donne, le défi pour Djibouti est l'élaboration d'une politique de prévention sur le long terme pour ne pas être pris de court et être réduit dans l'urgence à quémander l'aide internationale à la moindre dépression tropicale. Un plan de gestion de crise opérationnel, pour faire face 
à la survenue d'un autre épisode majeur, serait hautement souhaitable. Dans ce plan, un volet sur les évacuations des populations installées en zones inondables est à élaborer puis tester. Il est évident que l'effort doit se porter sur les deux communes sinistrées par Sagar. La mise en place d'un tel plan de gestion est un défi supplémentaire pour cette ville et plus largement pour l'ensemble la région.

\section{BIBLIOGRAPHIE}

Ayan M., Ozer P., 2013. RecentExtremePrecipitation and Temperature Changes in Djibouti City (1966-2011), Journal of Climatology [En ligne], vol. 2013, article ID 928501. DOI: https://doi.org/ $10.1155 / 2013 / 928501$

Banque mondiale, 2018. Rapport d'évaluation rapide des dommages et besoins, Post-cyclone Sagar. Djibouti, $33 \mathrm{p}$.

Chauvin F., Royer J.-F., 2008. Des cyclones et des hommes. La Météorologie, Météo et Climat, 8 e série, $n^{\circ} 61$, p. 52-66.

Deo A.A., Ganer D.W., 2015. Variability in Tropical Cyclone Activity Over Indian Seas in Changing Climate. International Journal of Science and Research, vol. 4, $\mathrm{n}^{\circ}$ 5, p. 880-886.

Evan A.-T., Camargo S.J., 2011. A Climatology of Arabian Sea Cyclonic Storms. Journal of climate [En ligne], $\mathrm{n}^{\circ}$ 24, p. 140-158. DOI: https://doi.org/10.1175/2010JCLI3611.1

Evan A.-T., Ramanathan V., 2011. Arabian Sea tropical cyclones intensified by emissions of black and other aerosols. Nature [En ligne], vol. 479, p. 94-97. DOI: https://doi.org/10.1038/nature10552

Camargo S., Sobel A., Barnston A., Emanuel K., 2007. Tropical cyclone genesis potential index in climate models. Tellus A: Dynamic Meteorology and Oceanography [En ligne], vol. 59, nº 4, p. 428-443. DOI: https://doi.org/10.1111/j.1600-0870.2007.00238.x

Hoarau K., Bernard J., Chalonge L., 2012. Intense tropical cyclone activities in the northern Indian Ocean. International Journal of Climatology [En ligne], $\mathrm{n}^{\circ}$ 32, p. 1935-1945. DOI: https://doi.org/ 10.1002/joc.2406

Hussain M.-A., Abbas S., Ansari R., 2011. Persistency analysis of cyclone history in Arabian sea. The Nucleus, vol. $48, \mathrm{n}^{\circ}$ 4. p. 273-277.

Kossin J.-P., Emanuel K.-A., Vecchi G.-A., 2014. The poleward migration of the location of tropical cyclone maximum intensity. Nature [En ligne], vol. 509, $\mathrm{n}^{\circ}$ 7500, p. 349-352. DOI: 10.1038/ nature 13278

Maneesha S., Manasa R.-B., 2015. Impact of SST on tropical cyclones in North Indian Ocean. 8th International Conference on Asian and Pacific Coasts (APAC 2015), Procedia Engineering , $\mathrm{n}^{\circ} 116$, p. 1072-1077.

Mrutyunjay M., Ajit T., 2011. Best track parameters of tropical cyclones over the North Indian Ocean: A review. Natural Hazards, vol. 63, n 3, p. 285-1317.

Nicholson S., 2013. The Predictability of Rainfall over the Greater Horn of Africa. Part I: Prediction of Seasonal Rainfall. Journal of hydrometeorology, vol. 15, p. 1011-1027. 
Nour Ayeh M., 2015. La ville de Djibouti entre intégration aux enjeux mondiaux et fragmentation urbaine. Territoire en mouvement Revue de géographie et aménagement [En ligne], $\mathrm{n}^{\circ} 27-28, \mathrm{p}$. 19. DOI: https://doi.org/10.4000/tem.3183

Nour Ayeh M., Ali Sougueh L., 2017. L'indice de chaleur pour évaluer la pénibilité du climat des villes littorales des zones arides : le cas de Djibouti. AIC, p. 523-531.

Omar Ali B., 2018. Risques naturels et développement dans la région de l'IGAD. Thèse de doctorat, université Paul Valéry-Montpellier 3, 531 p.

Roux F., 2012. Fureur des cieux, cyclones tropicaux et autres tempêtes. Paris, Ellipse, 283 p.

Secrétariat d'État chargé des Affaires Sociales, 2018. Évaluation de l'Impact Humain du cyclone Sagar. Djibouti, $58 \mathrm{p}$.

Singh O.P., Ali Khan T., Rahman S., 2001. Has the frequency of intense tropical cyclones increased over North Indian Ocean? Current Science, vol. 80, $n^{\circ} 4$, p. 575-580.

UNITAR, 2018. Flood analysis and evolution tropical cyclone Sagar [En ligne], 15 p. URL: https:// unitar.org/maps/map/2810

Webster P.J., Holland G.J., Curry J.A., Chang H.-R., 2005. Changes in Tropical Cyclone Number, Duration, and Intensity in a Warming Environment. Science [En ligne], vol. 309 n 5742 , p. 1844-1846. DOI: $10.1126 /$ science. 1116448

\section{NOTES}

1. $\mathrm{y}(\mathrm{x})=0.0110 \mathrm{x}+1.007$. Cf. Hussain et al., 2011.

2. La Météorologie, $8^{\mathrm{e}}$ série, $\mathrm{n}^{\circ} 18$, juin 1997.

3. http://www.meteo.nc/en-savoir-plus/comprendre-la-meteo/phenomenes-particuliers/faq/ 34-records/162-quelle-est-la-plus-grande-distance-parcourue-par-un-cyclone-tropical (source Météo France).

4. Établi par K. Emanuel en 2005.

5. Transformé en Ministère des Affaires Sociales et des Solidarités (MASS)

6. Devenue institut national de la statistique de Djibouti en 2019

7. Secrétariat d'État chargé des Affaires Sociales

8. Environ $10 \mathrm{M} \$(1 \$=177 \mathrm{DJF})$.

9. Office National d'Assistance aux Réfugiés et des Sinistrés.

\section{RÉSUMÉS}

Les régions du golfe d'Aden, qui connaissent une situation anticyclonique permanente, sont théoriquement en dehors des zones affectées par les cyclones tropicaux. Pourtant, l'analyse des données sur les quarante dernières années recense trente cyclones ou dépressions tropicales ayant touché la région. Fait plus remarquable, leur incidence semble avoir largement augmenté, tout comme leur intensité, sans que l'on sache clairement si cette recrudescence est à mettre au crédit du réchauffement climatique tant la machine climatique sur ce petit bassin est complexe. 
La faible capacité de résistance des pays de la région face à ces phénomènes météorologiques est révélée par l'impact du cyclone Sagar les 19 et 20 mai 2018 à Djibouti. Bien que rétrogradé en tempête tropicale, Sagar a coûté la vie à deux personnes et a inondé et impacté plus de $40 \%$ de la population de la capitale djiboutienne (soit plus de 200000 habitants). Cette catastrophe a clairement montré l'état d'impréparation d'une ville pauvre de climat aride face à ce type d'événement.

The Gulf of Aden regions, which are experiencing quite permanent high pressures, are theoretically outside the areas affected by tropical cyclones. However, the analysis of data over the past 40 years identifies 30 tropical cyclones or depressions that have affected the region. More remarkably, their impact seems to increase significantly, as does their intensity. However, it is not clear whether this upsurge is due to global warming as the climate mechanisms on this small basin are complex. The low resilience of countries in the region facing weather events is revealed by the impact of Cyclone Sagar on 19 and 20 May 2018 in Djibouti. Although downgraded to a tropical storm, Sagar triggered two fatalities. It flooded and damaged more than $40 \%$ of the population of the main town of Republic of Djibouti (i.e. more than 200,000 inhabitants). This disaster clearly shows the unpreparedness of such poor city under arid climate in developing countries.

\section{INDEX}

Keywords : Global warming, tropical storm, flood, urban vulnerability, Djibouti

Mots-clés : Réchauffement atmosphérique, cyclone tropical, inondation, vulnérabilité urbaine, Djibouti

Thèmes : Sur le Champ - Sur le Terrain

\section{AUTEURS}

\section{JEAN-PHILIPPE CHEREL}

Jean-Philippe Cherel, jean-philippe.cherel@univ-montp3.fr, est ingénieur de recherche, membre de l'UMR GRED, à l'Université Paul Valéry Montpellier. Il a notamment publié :

- Vinet F. et al., 2019. La cartographie des zones inondables : de l'événement prévu à l'événement réel. Retour d'expérience sur les inondations à Lamalou-les-Bains (sept. 2014). Géorisques, $\mathrm{n}^{\circ} 8$, p. 43-54.

- Omira R., Batista M.A., Leone F. et al., 2013. Performance of coastal sea-defense infrastructure at El Jadida (Morocco) against tsunami threat: lessons learned from the Japanese 11 March 2011 tsunami. Natural Hazards and Earth System Sciences [En ligne], vol. 13, n 7, p. 1779-1794. https:// www.nat-hazards-earth-syst-sci.net/13/1779/2013/nhess-13-1779-2013.html; DOI : https:// doi.org/10.5194/nhess-13-1779-2013

- Leone F. et al., 2012. Évaluation des vulnérabilités territoriales et humaines face aux tsunamis au Maroc (façade atlantique et ville d'El Jadida) : données historiques, modélisation de l'aléa et des enjeux humains, critères de vulnérabilité, indicateurs de risque, aide à la gestion des évacuations. Rapport WP5 (D5.4., D5.5, D5.6), Projet ANR-08-RISKNAT-05 MAREMOTI (MAREgraphie, observations de tsunaMis, mOdélisation et éTudes de vulnérabIlité pour le nord-est Atlantique et la Méditerranée occidentale), Université Montpellier 3-IRD, 190 p. + annexes. 


\section{BOUH OMAR ALI}

Bouh Omar Ali, bouh_omar_ali@univ.edu.dj, est maître de conférences à l'Université de Djibouti. Il a récemment publié :

- Omar Ali B., Vinet F., 2018. L'analyse spatiotemporelle de la mortalité des catastrophes naturelles dans la région de l'IGAD. Science et Environnement, $\mathrm{n}^{\circ} 32$.

- Omar Ali B., Vinet F., Cherel J.P, Le Martret H., 2016. Estimation de la vulnérabilité de la ville de DireDawa face aux crues de l'oued Dechatu (Éthiopie). Actes des Journées des doctorants de l'IM2E (Institut Montpellierains de l'Eau et de l'Environnement), 5 p. http://www.im2e.org/docs/jdd/ 2016/Oral/Bouh\%200mar\%20Ali_JDD_IM2E.pdf

- Omar Ali B., Vinet F. (2016), Évaluation de la vulnérabilité des ménages face au risque d'inondation dans la ville de Dire-Dawa (Éthiopie). Actes des $9^{\mathrm{e}}$ Journées fiabilité des matériaux et des structures, Université de Lorraine, $7 \mathrm{p}$.

\section{MOUSTAPHA NOUR AYEH}

Moustapha Nour Ayeh, moustapha_nour_ayeh@univ.edu.dj, est directeur des études en sciences humaines et sociales à l'Université de Djibouti. Il a récemment publié :

- Nour Ayeh M, 2019. La dynamique des températures et ses risques pour les populations de Djibouti dans le contexte du réchauffement global. NAAJ. Revue africaine sur les changements climatiques et les énergies renouvelables [En ligne], vol. 1, n 1, https:// www.revues.scienceafrique.org/naaj/texte/nour-ayeh2019/

- Nour Ayeh M, Ali Sougueh L, 2017. L'indice de chaleur pour évaluer la pénibilité du climat des villes littorales des zones arides : le cas de Djibouti. Association internationale de climatologie, Actes de colloque. p. 523-531.

- Nour Ayeh M, 2015. La ville de Djibouti entre intégration aux enjeux mondiaux et fragmentation urbaine. Territoire en mouvement Revue de géographie et aménagement [en ligne], n²7-28. http://journals.openedition.org/tem/3183 ; DOI : https://doi.org/10.4000/tem.3183

\section{FREDDY VINET}

Freddy Vinet, freddy.vinet@univ-montp3.fr, est professeur à l'Université Paul Valéry Montpellier et membre de l'UMR GRED. Il a récemment publié :

- Vinet F., Mathieu Peroche, Philippe Palany et al., 2020. Collecte et gestion des débris postcycloniques à Saint-Martin (Antilles françaises) après le passage du cyclone Irma (sept. 2017), Cybergeo : European Journal of Geography [En ligne], Environnement, Nature, Paysage, document 937. http://journals.openedition.org/cybergeo/34154; DOI https://doi.org/10.4000/cybergeo. 34154

- Tramblay Y, Mimeau L., Neppel L., Vinet F., Sauquet E., 2019. Detection and attribution of flood trends in Mediterranean basins Hydrology and Earth System Sciences [En ligne], vol. 23, $\mathrm{n}^{\circ} 11$. https://www.hydrol-earth-syst-sci.net/23/4419/2019/ ; DOI: https://doi.org/10.5194/ hess-23-4419-2019 - Vinet F. (ed.), 2018. Inondations. Vol. 1. La connaissance du risque et vol. 2. La gestion du risque. ISTE Press Elsevier. 